\title{
Multiplications in the Complex Bordism Theory with Singularities
}

\author{
Dedicated to Professor Ryoji Shizuma on his 60-th birthday
}

By

Nobuo ShIMADA and Nobuaki YAGITA*

\section{Introduction}

In 1967, D. Sullivan [13] introduced a bordism theory based on manifolds with singularities and successively N. Baas [3], [4] studied and reformulated the theory so that it has been given a more accessible ground.

This theory is considered as a natural generalization of usual bordism theory, and for each given singularity class $\mathscr{S}=\left\{P_{1}, P_{2}, \cdots\right\}$, a sequence of closed manifolds, one obtains such a theory. Thus there have appeared various interesting generalized homology theories.

For example, as Baas [4] shows, there exists a tower of homology theories and natural transformations connecting complex bordism to ordinary singular homology.

One of main problems with these theories has been to show whether they are multiplicative or not (Baas [6]). And the purpose of the present paper is to study this problem.

For convenience sake, we will restrict ourselves to the case of complex bordism theory $M U(\mathscr{S})_{*}(\quad)$ with singularity class $\mathscr{S}$. In this theory we introduce natural (external) multiplications (§3 and $\S 6$ )

$$
\begin{aligned}
\mu_{\boldsymbol{E}}: M U(\mathscr{S})_{a}(X, Y) \otimes M U(\mathscr{S})_{b}(V, W) \\
\quad \rightarrow M U(\mathscr{S})_{a+b}(X \times V, Y \times V \cup X \times W)
\end{aligned}
$$

which are "admissible" in an analogous sense of Araki-Toda [2], where $\boldsymbol{E}=\left\{E_{1}, E_{2}, \cdots\right\}$ means a sequence of Morara's manifolds $E_{i}$ one for each

Received February 23, 1976.

* Department of Mathematics, Tokyo Institute of Technology, Tokyo. 
$P_{i}(\S 2)$.

Although these multiplications $\mu_{\boldsymbol{E}}$ depend on $\boldsymbol{E}$ and primarily on $\mathscr{S}$, all of them are shown to be associative (Theorem 5.25).

As for commutativity we obtain an "obstruction" formula (Theorem 4.12). And for certain favoring cases where the obstructions vanish, $\mu_{\boldsymbol{E}}$ become commutative ( $\S 6)$. It follows, then, that the dual cohomology theories $M U(\mathscr{S})^{*}(\quad)$ are multiplicative and hence the representing spectra $M U$ $(\mathscr{S})$ become commutative ring spectra. Among them, we can show that, the "integral" Brown-Peterson spectrum BP for an odd prime $p$ (BrownPeterson [7]) is a ring spectrum (Corollary 6.7).

\section{Added after completion of the manuscript of this paper.}

In a recent issue of Izvestija Akad. Nauk SSSR, Ser. Mat. 39, No. 5 (1975), 1065-1092, the following paper appeared: O. K. Mironov, "Existence of multiplicative structures in the theories of cobordism with singularities" (in Russian).

Although our work has been done independently of his own, there may be some overlaps in the results.

Mironov dealt with the same objects as ours, but in more than one category of manifolds, i.e. with $S O, U, S U$ and $S p$ structure respectively. He used an inductive construction (on $n$ ) for defining multiplications in the bordism theories $\Omega_{*}{ }^{\Sigma_{n}}(\quad)$ with singularity type $\Sigma_{n}=\left\{P_{1}, \cdots, P_{n}\right\}$. ( $\sum_{n}$ correspond to our $\mathscr{S}_{n}$ ).

On this point his method seems considerably different from ours. As for commutativity and associativity, he treats only the case $n=1$, in the above paper, with detailed analysis for the $\bmod q$ theories.

\section{$\S$ 1. Baas-Sullivan Bordism Groups}

Recall first some notions concerning manifolds with singularities and the bordism theory based on such manifolds ([4]). Throughout this paper, by manifolds we mean compact, weakly complex manifolds with corners (see [8], [9] for manifolds with corners).

Definition 1.1. $V$ is a decomposed manifold of type $n$, iff there 
exist submanifolds $\partial_{0} V, \partial_{1} V, \cdots, \partial_{n} V$ of codimension zero of the boundary $\partial V$ such that

$$
\partial V=\partial_{0} V \cup \partial_{1} V \cup \cdots \cup \partial_{n} V
$$

where union means identification along common part of boundary.

Each $\partial_{i} V$ is again a decomposed manifold by defining

$$
\partial_{j}\left(\partial_{i} V\right)=\left\{\begin{array}{lll}
\partial_{j} V \cap \partial_{i} V & \text { for } & j \neq i \\
\phi & \text { for } & j=i
\end{array}\right.
$$

and we can continue defining $\partial_{k}\left(\partial_{j}\left(\partial_{i} V\right)\right)$ etc.

Let $\mathscr{S}_{n}=\left\{P_{1}, P_{2}, \cdots, P_{n}\right\}$ be a sequence of closed weakly complex manifolds of (real) even dimensions. We call it a singularity class (of weakly complex manifolds).

Definition 1.2. A manifold $A$ is called an $\mathscr{S}_{n}$-manifold, iff

i) to each ordered set $\omega=\left(i_{1}, \cdots i_{k}\right)$ of integers in $\{0,1, \cdots, n\}$, there associates a decomposed manifold (of type n) $A(\omega)$ such that

a) $A(\phi)=A$,

b) there exist isomorphisms (of weakly complex manifolds)

$$
\alpha(i ; \omega): \partial_{i} A(\omega) \approx \begin{cases}P_{i} \times A(i, \omega) & \text { for } \quad i \notin \omega \\ \phi & \text { for } \quad i \in \omega,\end{cases}
$$

where $P_{0}=*$ and $(i, \omega)$ denotes the ordered set $\left(i, i_{1}, \cdots, i_{k}\right) \subset\{0,1, \cdots, n\}$,

c) $A(\sigma(\omega))=\varepsilon(\sigma) A(\omega)$ for $\sigma \in S_{\omega}$,

here $S_{\omega}$ means the permutation group of the set $\omega$, and $\varepsilon(\sigma)$ the sign of $\sigma$, so that $-A(\omega)$ means the negative of $A(\omega)$, i.e. the manifold $A(\omega)$ with the opposite $U$-structure.

ii) for any $(i, j) \subset\{0,1, \cdots, n\}$ the following diagram commutes

$$
\begin{aligned}
& \partial_{j} \partial_{i} A(\omega) \stackrel{\alpha(i ; \omega) \mid \partial_{j}}{\longrightarrow} P_{i} \times \partial_{j} A(i, \omega) \stackrel{i d \times \alpha(j ;(i, \omega))}{\longrightarrow} P_{i} \times P_{j} \times A(j, i, \omega) \\
& i d \downarrow \\
- & \partial_{i} \partial_{j} A(\omega) \stackrel{\alpha(j ; \omega) \mid \partial_{i}}{\longrightarrow}-P_{j} \times \partial_{i} A(j, \omega) \stackrel{i d \times \alpha(i ;(j, \omega))}{\longrightarrow}-P_{j} \times P_{i} \times A(i, j, \omega)
\end{aligned}
$$


where $T$ is the twisting map and all of the maps are isomorphisms.

An $\mathscr{S}_{n}$-manifold may be denoted by $\{A(\omega), \alpha(i ; \omega)\}$, or simply by $A$, and the dimension of $\mathscr{S}_{n}$-manifold $A$ is defined to be that of the ambiant manifold $A(\phi)$.

Definition 1.3. An isomorphism $\varphi: A \rightarrow B$ between $\mathscr{S}_{n}$-manifolds $\{A(\omega), \alpha(i, \omega)\}$ and $\{B(\omega), \beta(i, \omega)\}$ is defined by a system of isomorphisms of weakly complex manifolds $\varphi(\omega): A(\omega) \rightarrow B(\omega)$ such that the following diagram commutes

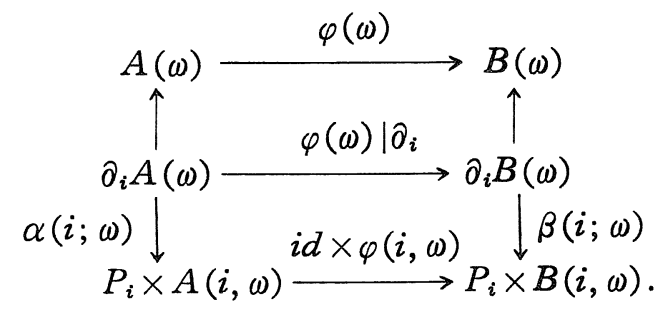

Let $(X, Y)$ be a pair of topological spaces.

Definition 1.4. A singular $\mathscr{S}_{n}$-manifold, $(A, f)$ in $(X, Y)$ is given by a system of pairs $\{(A(\omega), f(\omega))\}$ such that

i) $A=\{A(\omega), \alpha(i ; \omega)\}$ is an $\mathscr{S}_{n}$-manifold,

ii) $f(\omega): A(\omega) \rightarrow X$ are continuous maps such that the following diagrams commute

a)

$$
\begin{gathered}
A(\omega) \stackrel{f(\omega)}{\longrightarrow} X \supset Y \\
\left.\alpha(i ; \omega)\right|_{i} A(\omega) \\
P_{i} \times A(i, \omega) \stackrel{\text { pr. }}{\longrightarrow} A(i, \omega)
\end{gathered}
$$

b)

$$
\begin{aligned}
& A(\omega) \stackrel{f(\omega)}{i d \prod_{\varepsilon(\sigma) A(\sigma(\omega))}^{\longrightarrow}} X \\
& \int
\end{aligned}(\sigma(\omega))
$$


Definition 1.5. An isomorphism between singular $\mathscr{S}_{n}$-manifolds $(A, f)$ and $(B, g)$ in $(X, Y)$ is given by an isomorphism $\varphi: A \rightarrow B$ of $\mathscr{S}_{n}$-manifolds such that the following diagrams commute

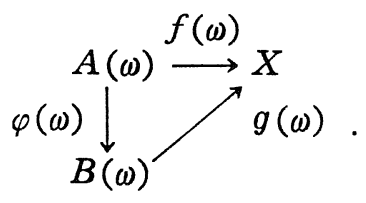

Definition 1.6. A singular $\mathscr{S}_{n}$-manifold $(A, f)$ in $(X, Y)$ bords, iff there exists a singular $\mathscr{S}_{n}$-manifold $(B, g)$ (we do not require $g(0, \omega)$ to factor through $Y$ ) such that $A(\omega)$ are submanifold of codimension zero of $B(\omega, 0)$ and

$$
g(\omega, 0) \mid A(\omega)=f(\omega), \quad g(\omega, 0)\left(B(\omega, 0)-A(\omega)^{\circ}\right) \subset Y
$$

where $A(\omega)^{\circ}$ denotes the interior of $A(\omega)$.

In particular, we define the "boundary" $\left(\partial_{0} A, \partial_{0} f\right)=(A(0), f(0))$ of a singular $\mathscr{S}_{n}$-manifold $(A, f)$ in $(X, Y)$ by

$$
\left(\partial_{0} A\right)(\omega)=A(\omega, 0),\left(\partial_{0} f\right)(\omega)=f(\omega, 0) .
$$

Then $\left(\partial_{0} A, \partial_{0} f\right)$ becomes a singular $\mathscr{S}_{n}$-manifold in $Y=(Y, \phi)$.

If $\partial_{0} A=\phi, A$ is called a closed $\mathscr{S}_{n}$-manifold.

Now for the formation and basic properties of the Baas-Sullivan bordism groups $M U\left(\mathscr{S}_{n}\right)_{*}(X, Y)$ we refer to [4]. We cite here the following facts which will be needed below.

First of all, we have

Proposition 1.8 (Theorem 3.3 in [4]). The functor $\operatorname{MU}\left(\mathscr{S}_{n}\right)_{*}$ ( ) forms a generalized homology theory.

The group $M U\left(\mathscr{S}_{n}\right)_{*}(X, Y)$ is considered as a (two-sided) $M U_{*}(p t)$ module in a canonical way, that is, in forming the usual product of a closed manifold and an $\mathscr{S}_{n}$-manifold.

For the next proposition, let $\mathscr{S}_{n-1}^{(i)}=\left\{P_{1}, \cdots, \widehat{P}_{i}, \cdots, P_{n}\right\}$ be the subsequence of $\mathscr{S}_{n}$ obtained by deleting the $i$-th singularity manifold $P_{i}$. For $i \in\{1,2, \cdots, n\}$, define a homomorphism of degree $p_{i}\left(p_{i}=\operatorname{dim} P_{i}\right)$ : 


$$
\beta_{i}: M U\left(\mathscr{S}_{n-1}^{(i)}\right)_{*}(X, Y) \rightarrow M U\left(\mathscr{S}_{n-1}^{(i)}\right)_{*}(X, Y)
$$

induced from the above multiplication by the element $\left[P_{i}\right]$ of $M U_{*}\left(p t_{0}\right)$, i.e.

$$
\beta_{i}[A, f]=\left[P_{i} \times A, f \circ p r\right]
$$

where $[A, f]$ denotes the bordism class of $(A, f)$. Further, let

$$
\gamma_{i}: M U\left(\mathscr{S}_{n-1}^{(i)}\right)_{*}(X, Y) \rightarrow M U\left(\mathscr{S}_{n}\right)_{*}(X, Y)
$$

be defined by regarding $\mathscr{S}_{n-1}^{(i)}$-manifold $A$ naturally as an $\mathscr{S}_{n}$-manifold $A$ with $\partial_{i} A=\phi$.

Finally the Bockstein operator of degree $-p_{i}-1$

$$
\delta_{i}: M U\left(\mathscr{S}_{n}\right)_{*}(X, Y) \rightarrow M U\left(\mathscr{S}_{n-1}^{(i)}\right)_{*}(X, Y)
$$

is defined by

$$
\delta_{i}[A, f]=[A(i), f(i)] .
$$

Then we have Bockstein exact sequence.

Proposition 1.12 (Theorem 3.2 in [4]). The sequence

$$
\begin{aligned}
& \cdots \longrightarrow M U\left(\mathscr{S}_{n-1}^{(i)}\right)_{*}(X, Y) \stackrel{\beta_{i}}{\longrightarrow} M U\left(\mathscr{S}_{n-1}^{(i)}\right)_{*}(X, Y) \stackrel{\gamma_{i}}{\longrightarrow} M U\left(\mathscr{S}_{n}\right)_{*}(X, Y) \\
& \stackrel{\delta_{i}}{\longrightarrow} M U\left(\mathscr{S}_{n-1}^{(i)}\right)_{*}(X, Y) \stackrel{\beta_{i}}{\longrightarrow \cdots}
\end{aligned}
$$

is exact.

For the coefficient group, we have

Proposition 1.13 (Theorem 4.1 in $[4]$ ). If the sequence $\left\{\left[P_{1}\right], \cdots\right.$, $\left.\left[P_{n}\right]\right\}$ of the bordism classes $\left[P_{i}\right], P_{i} \in \mathscr{S}_{n}$, constitutes a regular sequence in $M U_{*}(p t)$, we have an isomorphism

$$
\operatorname{MU}\left(\mathscr{S}_{n}\right)_{*}(p t) \approx M U_{*}(p t) /\left(\left[P_{1}\right], \cdots,\left[P_{n}\right]\right)
$$

as an $M U_{*}(p t)$-module, where the right hand side means the quotient algebra of $M U_{*}(p t)$ by the ideal generated by $\left[P_{1}\right], \cdots,\left[P_{n}\right]$.

Notations. The above homomorphisms $\beta_{i}, \gamma_{i}$ and $\delta_{i}$ will be some- 
times denoted by $\beta_{P_{i}}, \gamma_{P_{i}}$ and $\delta_{P_{i}}$ respectively, and the notations $\delta_{i_{1} \ldots i_{k}}$ $=\delta_{i_{1}} \delta_{i_{2}} \cdots \delta_{i_{k}}, \delta_{i_{1} \cdots i_{k}} A=A\left(i_{1} \cdots i_{k}\right)=A\left(P_{i_{1}} \cdots P_{i_{k}}\right)$ will be also used.

Remark. If singularity manifolds $Q_{1}, \cdots, Q_{n}$ are bordant to $P_{1}, \cdots, P_{n}$ respectively, then the corresponding groups $M U\left(Q_{1}, \cdots, Q_{n}\right)_{*}(\quad)$ and $M U$ $\left(P_{1}, \cdots, P_{n}\right)_{*}(\quad)$ are isomorphic.

\section{$\S 2$. Some Geometric Constructions and Basic Lemmas}

Recall first a geometric technique due to Morava (cf. Johnson-Wilson [10], Appendix).

Let $P$ be a closed, weakly complex manifold of dimension $p$. Put

$$
E(0)=I \times P \times P
$$

where $I$ denotes the unit interval $[0,1]$. This manifold $E(0)$ is to be given the canonical weakly complex structure induced from that of $P \times P$.

Define

$$
\begin{aligned}
& \partial_{1} E(0)=\partial E(0)=-(0 \times P \times \underline{P}) \cup(1 \times \underline{P} \times P), \\
& E(10)=-(0 \times P) \cup(1 \times P), \varepsilon(1 ; 0): \partial_{1} E(0) \approx \underline{P} \times E(10),
\end{aligned}
$$

where $\varepsilon(1 ; 0)$ denotes the twisting isomorphism defined by

$$
\hat{\varepsilon}(1 ; 0)(i, p, q)= \begin{cases}(q, 0, p) & \text { for } \quad i=0 \\ (p, 1, q) & \text { for } \quad i=1 .\end{cases}
$$

Here we have used the convention $\partial I=(-0) \cup(1)$.

Then $E(0)$ is a closed $\{P\}$-manifold of dimension $2 p+1$. Since $M U$ $(P)_{*}(p t) \approx M U_{*}(p t) /([P])$ (Proposition 1.11) and the odd dimension part of this group is zero, we can choose a $\{P\}$-manifold $E$ with $\partial_{0} E=E(0)$.

We will call such a manifold $E$ Morava's manifold for $P$ and sometimes denote it by $E_{P}$.

Lemma 2.2. Let $E$ and $E^{\prime}$ be Morava's manifolds for a fixed $P$. Then $E$ and $E^{\prime}$ determine a closed $\{P\}$-manifold $b\left(E, E^{\prime}\right)=E \cup_{i d}$ $\left(-E^{\prime}\right)$ representing an element $\beta\left(E, E^{\prime}\right)$ of $M U(P)_{2 p+2}(p t)$. Conversely, for any element $\beta$ of $M U(P)_{2 p+2}(p t)$ and any choice of Morava's manifold $E$ for $P$, there exists another $E^{\prime}$ such that $\beta\left(E, E^{\prime}\right)=\beta$. 
Proof. By $E \cup_{i d}\left(-E^{\prime}\right)$ we mean the manifold obtained from $E \cup$ ( $\left.-E^{\prime}\right)$ by identifying the part $E(0)$ with the corresponding part $E^{\prime}(0)$. The first half of the lemma is obvious. For the second half, we note that $\beta(E, E)=0$. For a given $\beta$, choose a closed manifold $B$, of which image in $M U_{*}(p t) /([P])$ gives $\beta$. Take $E \cup(-B)$ as $E^{\prime}$, then we have $\beta\left(E, E^{\prime}\right)=\beta$.

Now we will introduce several lemmas which are needful for our purpose.

These lemmas aim to analyse the groups $M U\left(\mathscr{S}_{n}\right)_{*}(X, Y)$, especially for the case of singularity class $\mathscr{S}_{n}$ having duplicated members.

Let $\mathscr{S}_{n}=\left\{P_{1}, \cdots, P_{n}\right\}$ be a sequence of closed manifolds as before, and suppose $P_{i}=P_{j}=P$ for some pair $(i, j), 1 \leqq i<j \leqq n$. Let $\mathscr{S}_{n-1}^{(i)}=\left\{P_{1}\right.$, $\left.\cdots, \widehat{P}_{i}, \cdots P_{n}\right\}$ be the subsequence $\mathscr{S}_{n}-\left\{P_{i}\right\}$.

Utilizing Morava's manifold $E$ for $P$, we shall define a homomorphism of degree $p+1$

$$
s_{i j}=s_{i j}^{E}: M U\left(\mathscr{S}_{n-1}^{(i)}\right)_{*}(X, Y) \rightarrow M U\left(\mathscr{S}_{n}\right)_{*}(X, Y)
$$

as follows. Given a singular $\mathscr{S}_{n-1}^{(i)}$-manifold $(B, g)$ in $(X, Y)$, and put

$$
s_{i j}(B)=I \times P \times B \bigcup_{j 0} E \times B(j)
$$

with identification of isomorphic parts of boundaries:

$$
\begin{array}{llc}
I \times P \times \partial_{j} B & & \partial_{0} E \times B(j) \\
& \approx \downarrow 1 \times 1 \times \beta(j) & \\
I \times P \times P_{j} \times B(j) \stackrel{i d}{\longrightarrow} & I \times P \times P \times B(j),
\end{array}
$$

here it should be noticed that we will make a convenience of labelling parts of $\partial B$ as if $B$ were an $\mathscr{S}_{n}$-manifold with $\partial_{i} B=\phi$. Thus $\partial_{j}, B(j)$ mean $\partial_{P_{j}}, B\left(P_{j}\right)$ respectively (See $\S 1$ ). Then $s_{i j}(B)$ is regarded as an $\mathscr{S}_{n}$-manifold by defining

$$
\partial_{k} s_{i j}(B)= \begin{cases}-0 \times P \times B & \text { for } k=i \\ 1 \times P \times B \bigcup_{j 0} \partial_{1} E \times B(j) & \text { for } k=j \\ -I \times P \times \partial_{k} B \bigcup_{j 0} E \times \partial_{k} B(j) & \text { for } k \neq i, j,\end{cases}
$$




$$
s_{i j}(B)(\omega)= \begin{cases}-I \times P \times B(\omega) \cup E \times B(\omega, j) & \text { for } \omega \not i, j \\ -\varepsilon(\sigma)\left(0 \times B\left(\omega^{\prime}\right)\right) & \text { for } \sigma(\omega)=\left(\omega^{\prime}, i\right) \\ \varepsilon(\tau)\left(1 \times B\left(\omega^{\prime \prime}\right) \cup(-1)^{\left|\omega^{\prime \prime}\right|} E(1) \times B\left(\omega^{\prime \prime}, j\right)\right. \\ & \text { for } \tau(\omega)=\left(\omega^{\prime \prime}, j\right), \\ & \left|\omega^{\prime \prime}\right|=\text { length of } \omega^{\prime \prime},\end{cases}
$$

$s_{i j}(g) \mid I \times P \times B=g \circ$ proj.

$s_{i j}(g) \mid E \times B(j)=g(j) \circ$ proj.

Inspection shows this is well-defined. .

The following lemma follows directly from $(2 \cdot 4)$.

Proposition 2.5. Let $\mathscr{S}_{n}$ and $\mathscr{S}_{n-1}^{(i)}$ be as above, and suppose $P_{i}=P_{j}=P$ for some $j, j>i . \quad P u t p=\operatorname{dim} P$. Then the Bockstein long exact sequence (Proposition 1.12) breaks in split exact sequences

$$
\begin{gathered}
0 \longrightarrow M U\left(\mathscr{S}_{n-1}^{(i)}\right)_{l}(X, Y) \stackrel{\gamma_{i}}{\longrightarrow} M U\left(\mathscr{S}_{n}\right)_{l}(X, Y) \\
\stackrel{\delta_{i j}^{E}}{\rightleftarrows} M U\left(\mathscr{S}_{n-1}^{(i)}\right)_{l-p-1}(X, Y) \longrightarrow 0
\end{gathered}
$$

such that $\delta_{i} s_{i j}=-i d$.

This means, in particular, that the homomorphism $\beta_{i}$ in Proposition 1.12 vanishes for the present case. But, this implies, in turn,

Corollary 2.6 (2.4 in [10]). For any singularity class $\mathscr{S}_{n}=\left\{P_{1}\right.$, $\left.\cdots, P_{n}\right\}$, the group $M U\left(\mathscr{S}_{n}\right)_{*}(X, Y)$ is a module over the quotient ring $M U_{*}(p t) /\left(\left[P_{1}\right], \cdots,\left[P_{n}\right]\right)$. The module structure is induced from the usual action of $M U_{*}(p t)$ (cf. Proposition 1.13).

Proposition 2.7. Under the same assumption as in Proposition 2.5, we have

$$
\delta_{j} \gamma_{i}=\gamma_{i} \hat{o}_{j} \text { for } i \neq j, \quad s_{i j} \gamma_{k}=\gamma_{k} s_{i j} \text { for } k \neq i, j
$$


ii)

$$
\delta_{k} s_{i j}^{E}= \begin{cases}-i d & \text { for } k=i \\ (i j)^{*} & \text { for } k=j \\ -s_{i j}^{E} \delta_{k} & \text { for } k \neq i, j,\end{cases}
$$

where

$$
(i j)^{*}: M U\left(\mathscr{S}_{n-1}^{(i)}\right)_{*}(X) \rightarrow M U\left(\mathscr{S}_{n-1}^{(j)}\right)_{*}(X)
$$

means the isomorphism induced by the transposition (ij) on the labels, and hence, by the exchange of $P_{j}$ for $P_{i}, \partial_{j}$ for $\partial_{i}$ and etc. $(c f .(2 \cdot 12))$

Proof. The equalities are directly seen from $(2 \cdot 4)$, except for the case $k=j$ in ii). For this case, we observe from $(2 \cdot 4)$

$$
\delta_{j} s_{i j}(B)=s_{i j}(B)(j)=1 \times B \bigcup_{j 0} E(1) \times B(j) .
$$

It will be sufficient to prove that $(i j)^{*} B \sim s_{i j}(B)(j)$ as $\mathscr{S}_{n-1}^{(j)}$-manifolds.

First note that $\partial E(1)=(0 \times P) \cup-(1 \times P)$, hence $E(1)$ is a $\{P\}$ manifold of odd dimension in virtue of the assumption $\operatorname{dim} P=$ even $(\S 1)$.

Let $I_{1}$ be a copy of the unit interval $[0,1]$. Consider $E(1) \cup I_{1} \times P$ with identification $\partial E(1) \equiv-\partial\left(I_{1} \times P\right) \quad(c f .(2 \cdot 1))$. Then this is a closed manifold representing an element of $M U_{\text {odd }}(p t)=0$. Therefore there exists a manifold $L$ such that $\partial L=E(1) \cup I_{1} \times P$.

Construct

$$
V=[0, \varepsilon]_{2} \times B \cup-L \times B(j)
$$

with identification of isomorphic parts of boundaries:

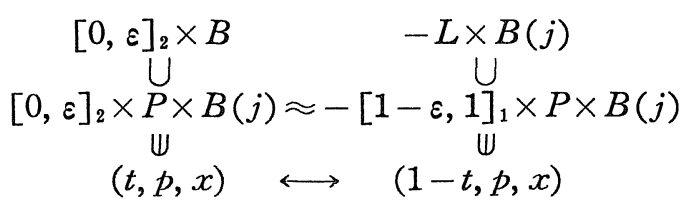

where we assume $0<\varepsilon<1-\varepsilon$.

Put

$$
\begin{aligned}
& \partial_{0} V=-\left(0_{2} \times B \cup E(1) \times B(j)\right) \Perp\left(\varepsilon_{2} \times B \cup-[\varepsilon, 1-\varepsilon]_{1} \times P \times B(j)\right), \\
& \partial_{i} V=-[0, \varepsilon]_{1} \times P \times B(j), \quad \partial_{j} V=\phi, \\
& \partial_{k} V=-\left([0, \varepsilon]_{2} \times \partial_{k} B \cup L \times \partial_{k} B(j)\right) \quad \text { for } \quad k \neq 0, i, j,
\end{aligned}
$$


where $\Perp$ means disjoint union.

We would like to omit to give the explicit forms of $V(\omega)$.

Thus $V$ is an $\mathscr{S}_{n-1}^{(j)}$-manifold and

$$
\partial_{0} V=-s_{i j}(B)(j)+(i j) * B,
$$

which was to be shown.

For the next proposition, we insert a small construction and a lemma.

Chose a Morava's manifold $E=E_{P}$ for a closed manifold $P$.

Define

$$
\begin{aligned}
& \varphi: I=[0,1] \rightarrow I, \quad \text { by } \varphi(t)=1-t, \\
& T: P \times P \rightarrow P \times P, \quad \text { by } T(p, q)=(q, p) .
\end{aligned}
$$

Then we have an isomorphism

$$
\varphi \times T: E(0)=I \times P \times P \approx-E(0)
$$

and we construct a closed $\{P\}$-manifold

$$
a(E)=E \bigcup_{\varphi \times T} E
$$

from two copies of $E$, identifying the parts $E(0)$ 's by the isomorphism $(2 \cdot 9)^{\prime}$.

The following lemma follows easily.

Lemma 2.11. Let $E$ and $E^{\prime}$ be Morava's manifolds for the same P. Then, we have

$$
\alpha(E)-\alpha\left(E^{\prime}\right)=2 \beta\left(E, E^{\prime}\right), \alpha(E)=[a(E)],
$$

in $M U(P)_{*}(p t)$. (See Lemma 2.2 for the definition of $\beta\left(E, E^{\prime}\right)$.)

Let now $\mathscr{S}_{n}$ be as before and assume $P_{i}=P_{j}=P, i<j$, for some $i, j$ in $\{1,2, \cdots, n\}$. Let $\sigma=(i j)$ be the transposition. This induces a canonical isomorphism

$$
(\underline{i j})^{*}: \operatorname{MU}\left(\mathscr{S}_{n}\right)_{*}(\quad) \rightarrow \operatorname{MU}\left(\sigma\left(\mathscr{S}_{n}\right)\right)_{*}(\quad),
$$

where $\sigma\left(\mathscr{S}_{n}\right)=\left\{P_{1}, \cdots \stackrel{i}{P}_{j} \cdots \stackrel{j}{P}_{i} \cdots P_{n}\right\}$. Explicitly, for a singular $\mathscr{S}_{n}$-manifold $(A, f)$ it gives 


$$
(A, f) \mapsto\left(A^{*}, f^{*}\right)=\left(\underline{\sigma}^{*} A, \underline{\sigma}^{*} f\right),
$$

$\partial_{i} A^{*}=\partial_{\sigma(i)} A, A^{*}(\omega)=A(\sigma(\omega))$ and $f^{*}(\omega)=f(\sigma(\omega))$ etc.

Composing this isomorphism $(\underline{i j})^{*}$ with the trivial identification.

$$
\text { ८: } \operatorname{MU}\left(\sigma\left(\mathscr{S}_{n}\right)\right)_{*}(\quad) \equiv M U\left(\mathscr{S}_{n}\right)_{*}(\quad)
$$

induced from the obvious identification $\sigma\left(\mathscr{S}_{n}\right) \equiv \mathscr{S}_{n}$, we have an involution

$$
\bar{\sigma}^{*}=(\overline{i j})^{*}=\ell \circ(\underline{i j})^{*}: M U\left(\mathscr{S}_{n}\right)_{*}(\quad) \rightarrow M U\left(\mathscr{S}_{n}\right)_{*}(\quad) .
$$

Then the following proposition is one of key lemmas in our method.

Proposition 2.13. Under the above assumption on $\mathscr{S}_{n}$, we have

$$
\delta_{k}(\overline{i j}) *= \begin{cases}(j i) * \delta_{j} & \text { for } k=i \\ (i j) * \delta_{i} & \text { for } k=j \\ (\overline{i j}) * \delta_{k} & \text { for } k \neq i, j,\end{cases}
$$

ii)

$$
(\overline{i j})^{*}-1=s_{i j}^{E} \delta_{i}\left(1-(\overline{i j})^{*}\right)+\alpha\left(E_{j}\right) \times \delta_{i} \delta_{j}
$$

on $M U\left(\mathscr{S}_{n}\right)_{*}(\quad)$. More explicitly, ii) means

$$
\begin{aligned}
& \left(A^{*}, f^{*}\right)-(A, f) \sim \\
& \quad s_{i j}^{E}(A(i), f(i))-s_{i j}^{E}\left(A^{*}(i), f^{*}(i)\right)+\left(a\left(E_{j}\right) \times A(i j), f(i j) \circ p r\right)
\end{aligned}
$$

for a singular $\mathscr{S}_{n}$-manifold $(A, f)$, where the last term means the singular $\mathscr{S}_{n}$-manifold defined by

$$
\left.a\left(E_{j}\right) \times A(i j) \stackrel{\partial_{j}}{\longrightarrow} \partial_{1} a(E) \times A(i j) \quad \text { (cartesian product }\right)
$$

In this proposition the assertion i) is easily verified from the definitions of the terms involved there. (See $(1 \cdot 11),(2 \cdot 8)$ and $(2 \cdot 12)$ ".) For the proof of the assertion ii), we have to prepare some lemmas.

Lemma 2.14. Under the same situation as in Proposition 2.13, we have

$$
(\overline{i j}) * A-A \sim I \times\left(\partial_{i} A \cup \partial_{j} A\right),
$$


where the right hand side means the $\mathscr{S}_{n}$-manifold defined by

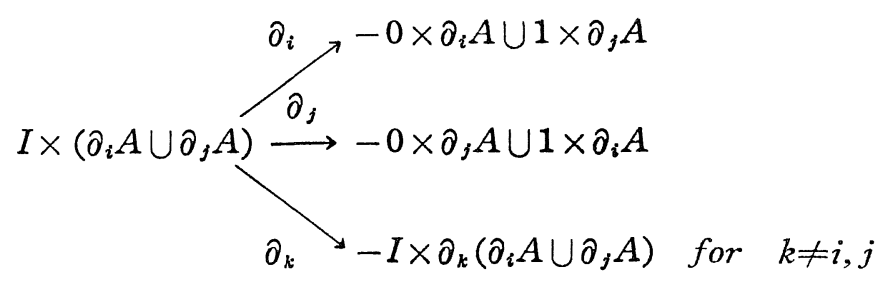

Proof. First replace $A$ with $A^{\prime}=A \cup[0, \varepsilon] \times \partial A$, so that $A^{\prime}$ has a collared neighborhood of boundary and itself is isomorphic to $A$. Construct $I \times A^{\prime}$ as an $\mathscr{S}_{n}$-manifold:

$$
\begin{gathered}
\left.\times\left(A \cup[0, \varepsilon] \times \partial_{0} A\right)\right\} \cup-I \times \varepsilon \times\left(\partial_{i} A \cup \partial_{j} A\right) \\
-0 \times[0, \varepsilon] \times \partial_{i} A \cup 1 \times[0, \varepsilon] \times \partial_{j} A
\end{gathered}
$$

Then, by inspection we see that $(-A) \cup(\overline{i j}) * A \cup\left(-I \times\left(\partial_{i} A \cup\right.\right.$ $\left.\partial_{j} A\right)$ ) bords $I \times A^{\prime}$ (See Definition 1.6).

Lemma 2.15. Under the same assumption as above, we have

$$
I \times\left(\partial_{i} A \cup \partial_{j} A\right) \sim s_{i j}^{E}(A(i))+s^{\prime}(A(j))+a\left(E_{j}\right) \times A(i j)
$$

where

$$
s^{\prime}(A(j))=I \times P \times A(j) \underset{\partial_{i}(\varphi \times 1) \partial_{0}}{\bigcup} E \times A(j i)
$$

is defined by identification

$$
-I \times P \times P \times A(i j) \stackrel{\varphi \times 1 \times 1}{\underset{ }{\longrightarrow}} E(0) \times A(j i)
$$

and it is considered as an $\mathscr{S}_{n}$-manifold: 


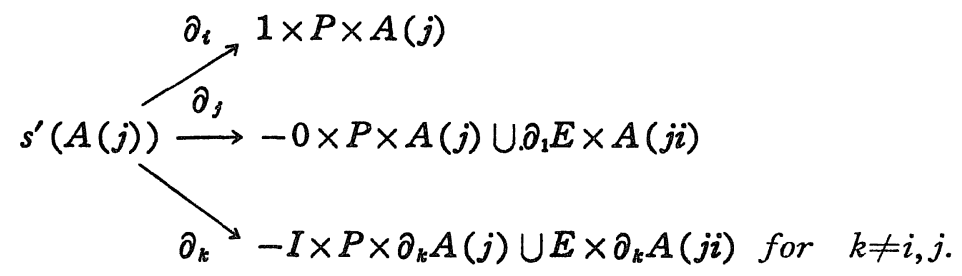

Proof. Since the detailed proof is considerably tedious, we will only outline the proof. Let $I_{1}$ and $I_{2}$ be copies of the unit interval $[0,1]$, and let $E^{\prime}$ and $E^{\prime \prime}$ be copies of Morava's manifold $E_{P}$.

Construct a "manifold"

$$
W=I_{1} \times I_{2} \times\left(\partial_{i} A \cup \partial_{j} A\right) \cup I_{3} \times\left(E^{\prime} \bigcup_{\varphi \times T} E^{\prime \prime}\right) \times A(i j)
$$

with identification:

$$
1_{1} \times I_{2} \times P \times P \times A(j i) \equiv 0_{3} \times I \times \partial_{j} \partial_{i} A \quad-0_{3} \times E^{\prime}(0) \times A(i j)
$$

Exactly speaking, this could not be called a manifold. In order to obtain a genuine manifold, we need a certain "thickening" process in the above attachment $(2 \cdot 18)$.

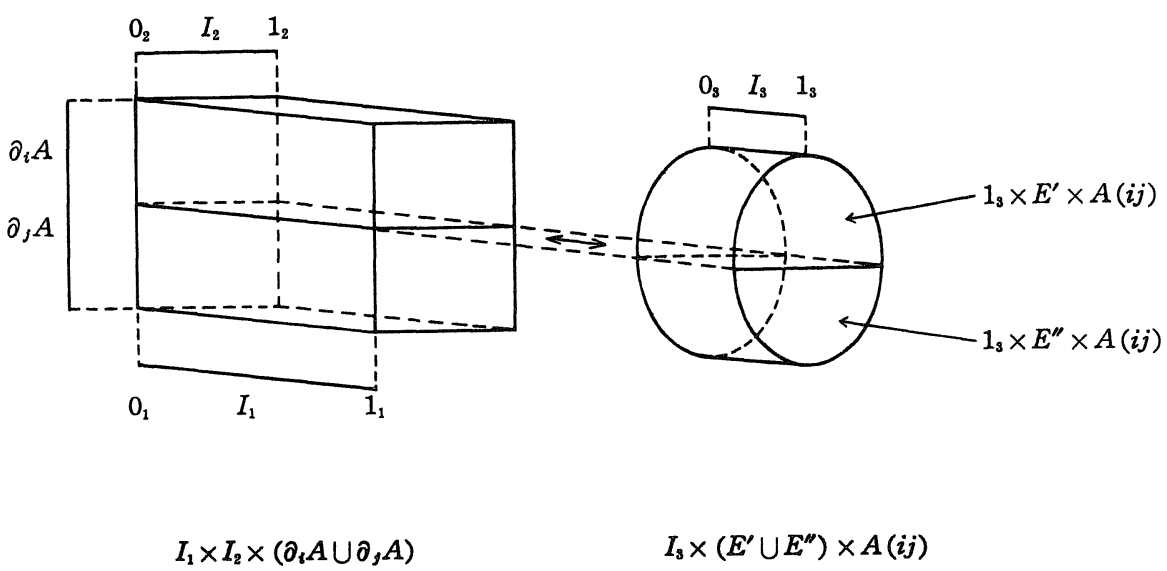

Figure 1 
But, for simplicity's sake, we shall content with the pseudo-manifold $W$. Now $W$ is considered as an $\mathscr{S}_{n}$ "“manifold" as follows:

$$
\begin{aligned}
\partial_{0} W= & I_{1} \times I_{2} \times \partial_{0}\left(\partial_{i} A \cup \partial_{j} A\right) \cup-0_{1} \times I_{2} \times\left(\partial_{i} A \cup \partial_{j} A\right) \\
& \cup\left(1_{1} \times I_{2} \times \partial_{i} A \cup-0_{3} \times E^{\prime} \times A(i j)\right) \cup\left(1_{1} \times I_{2} \times \partial_{j} A \cup\right. \\
& \left.-0_{3} \times E^{\prime \prime} \times A(i j)\right) \cup 1_{3} \times\left(E^{\prime} \cup E^{\prime \prime}\right) \times A(i j) \\
\partial_{i} W= & I_{1} \times 0_{2} \times \partial_{i} A \cup-I_{1} \times 1_{2} \times \partial_{j} A \\
\partial_{j} W= & I_{1} \times 0_{2} \times \partial_{j} A \cup-I_{1} \times 1_{2} \times \partial_{j} A \cup-I_{3} \times \partial_{1}\left(E^{\prime} \cup E^{\prime \prime}\right) \times A(i j) \\
\partial_{k} W= & I_{1} \times I_{2} \times \partial_{k}\left(\partial_{i} A \cup \partial_{j} A\right) \quad \text { for } k \neq 0, \mathrm{i}, \mathrm{j} .
\end{aligned}
$$

And there are isomorphisms:
a) $s_{i j}^{E}(A(i)) \approx 1_{1} \times I_{2} \times \partial_{i} A \cup 0_{3} \times E^{\prime} \times A(j i) \subset \partial_{0} W$
b) $s^{\prime}(A(j)) \approx 1_{1} \times I_{2} \times \partial_{j} A \cup 0_{3} \times E^{\prime \prime} \times A(j i) \subset \partial_{0} W$
c) $a\left(E_{j}\right) \times A(i j) \approx 1_{3} \times\left(E^{\prime} \bigcup_{\varphi \times T} E^{\prime \prime}\right) \times A(i j) \subset \partial_{0} W$.

In fact, the involved identifications will be seen in the following commutative diagram

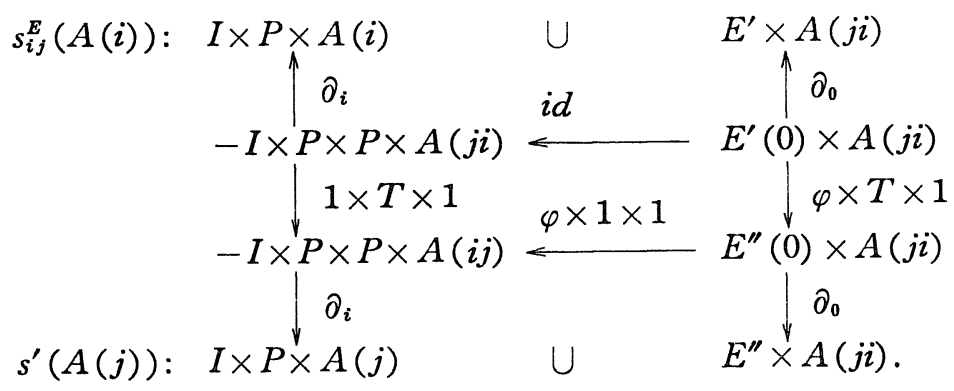

Then Lemma 2.15 follows easily.

Lemma 2.20. In the above lemma, we have an isomorphism

$$
s^{\prime}(A(j)) \approx-s_{i j}^{E}\left(A^{*}(i)\right)
$$

where $A^{*}=(\overline{i j}) * A$.

Proof. Define the following map between $s^{\prime}(A(j))$ and $-s_{i j}^{E}\left(A^{*}\right.$ (i)) (cf. $(2 \cdot 4),(2 \cdot 16),(2 \cdot 17))$ : 


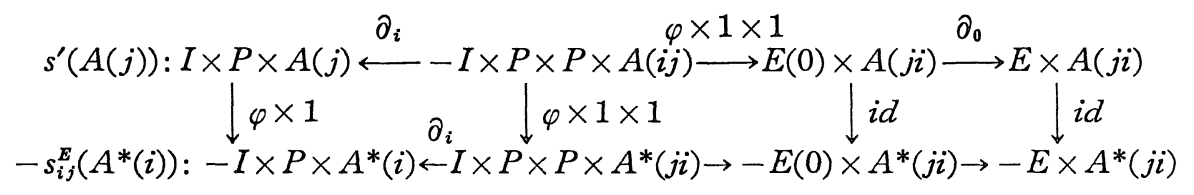

This map gives the isomorphism in the lemma.

Proof of Proposition 2.13, ii). Lemma 2.14, 2.15 and 2.20 give the desired proof.

\section{§ 3. Multiplication in $M U\left(\mathscr{S}_{n}\right)_{*}($ )}

Let $\mathscr{S}_{n}=\left\{P_{1}, \cdots, P_{n}\right\}$ and $\mathscr{I}_{m}=\left\{Q_{1}, \cdots, Q_{m}\right\}$ be singularity classes as defined in $\S 1$. Recall that all $P_{i}$ and $Q_{j}$ are closed, weakly complex manifolds of even dimensions.

Let $(A, f)$ be a singular $\mathscr{S}_{n}$-manifold in $(X, Y)$, and $(B, g)$ a singular $\mathscr{I}_{m}$-manifold in $(V, W)$. Put $a=\operatorname{dim} A$ and $b=\operatorname{dim} B$. Denote by $\mathscr{S}_{n}+\mathscr{I}_{m}=\left\{P_{1}, \cdots, P_{n}, Q_{1}, \cdots, Q_{m}\right\}$ the sequence obtained by juxtaposing $\mathscr{S}_{n}$ and $\mathscr{I}_{m}$ in their proper orders (cf. [3]).

Definition 3.1. The cross product $(A, f) \times(B, g)$ is defined as the singular $\left(\mathscr{S}_{n}+\mathscr{I}_{m}\right)$-manifold $(A \times B, f \times g)$ in $(X \times V, Y \times V \cup X \times W)$, which is given by the following data:

i) $\partial_{i}(A \times B)= \begin{cases}\partial_{0} A \times B \cup(-1)^{a} A \times \partial_{0} B & \text { for } i=0 \\ \partial_{i} A \times B & \text { for } 1 \leqq i \leqq n \\ (-1)^{a} A \times \partial_{i-n} B & \text { for } n+1 \leqq i \leqq n+m,\end{cases}$

ii) $\quad(A \times B)\left(\omega^{\prime}, \bar{\omega}^{\prime \prime}\right)=(-1)^{a_{\left|\omega^{\prime \prime}\right|}} A\left(\omega^{\prime}\right) \times B\left(\omega^{\prime \prime}\right)$

and

$$
\begin{aligned}
(A \times B) & \left(\omega^{\prime}, \bar{\omega}^{\prime \prime}, 0\right)=(-1)^{a\left|\omega^{\prime \prime}\right|}\left(A\left(\omega^{\prime}, 0\right)\right. \\
& \left.\times B\left(\omega^{\prime \prime}\right) \cup(-1)^{a} A\left(\omega^{\prime}\right) \times B\left(\omega^{\prime \prime}, 0\right)\right) \\
& \text { for } \omega^{\prime} \subset\{1, \cdots, n\}, \omega^{\prime \prime}=\left(j_{1}, \cdots, j_{l}\right) \subset\{1, \cdots, m\},\left|\omega^{\prime \prime}\right|=l, \\
& \quad \bar{\omega}^{\prime \prime}=\left(n+j_{1}, \cdots, n+j_{l}\right), \\
\text { iii }) \quad( & f \times g)\left(\omega^{\prime}, \bar{\omega}^{\prime \prime}\right)=f\left(\omega^{\prime}\right) \times g\left(\omega^{\prime \prime}\right)
\end{aligned}
$$


and

$$
(f \times g)\left(\omega^{\prime}, \bar{\omega}^{\prime \prime}, 0\right)=\left\{\begin{array}{lll}
f\left(\omega^{\prime}, 0\right) \times g\left(\omega^{\prime \prime}\right) & \text { on } & A\left(\omega^{\prime}, 0\right) \times B\left(\omega^{\prime \prime}\right) \\
f\left(\omega^{\prime}\right) \times g\left(\omega^{\prime \prime}, 0\right) & \text { on } & A\left(\omega^{\prime}\right) \times B\left(\omega^{\prime \prime}, 0\right)
\end{array}\right.
$$

for $\omega^{\prime}, \omega^{\prime \prime}, \bar{\omega}^{\prime \prime}$ as above.

Remark. We may also take $\left\{P_{1}, Q_{1}, P_{2}, Q_{2}, \cdots, P_{k}, Q_{k}, \cdots\right\}$ as $\mathscr{S}_{n}+\mathscr{I}_{m}$. In this case, the above definition 3.1 should receive a change of labelling in $\partial_{i}, \delta_{i}$ etc.

The cross product induces a bilinear map

$$
\begin{aligned}
\times: M U & \left(\mathscr{S}_{n}\right)_{a}(X, Y) \times M U\left(\mathscr{I}_{m}\right)_{b}(V, W) \\
& \rightarrow M U\left(\mathscr{S}_{n}+\mathscr{I}_{m}\right)_{a+b}(X \times V, Y \times V \cup X \times W) \\
& {[A, f] \times[B, g]=[(A, f) \times(B, g)] }
\end{aligned}
$$

or a linear map

$$
\begin{aligned}
\times & : M U\left(\mathscr{S}_{n}\right)_{a}(X, Y) \otimes M U\left(\mathscr{I}_{m}\right)_{b}(V, W) \\
& \rightarrow M U\left(\mathscr{S}_{n}+\mathscr{I}_{m}\right)_{a+b}(X \times V, Y \times V \cup X \times W)
\end{aligned}
$$

which are natural and compatible with the multiplication by elements of $M U_{*}(p t)$.

Moreover the cross product is apparently associative:

$$
\{(A, f) \times(B, g)\} \times(C, h)=(A, f) \times\{(B, g) \times(C, h)\} .
$$

Hereafter, we consider the case of $\mathscr{I}_{m}=\mathscr{S}_{n}$.

For convenience, denote by $\mathscr{S}_{n}^{\prime}=\left\{P_{1}^{\prime}, \cdots, P_{n}^{\prime}\right\}$ a copy of $\mathscr{S}_{n}$ so that $P_{i}^{\prime}=P_{i}$ for $i=1,2, \cdots, n$.

Then we have endomorphisms

$$
\pi_{12}^{P_{i}}: M U\left(\mathscr{S}_{n}+\mathscr{S}_{n}{ }^{\prime}\right)_{*}(\quad) \rightarrow M U\left(\mathscr{S}_{n}+\mathscr{S}_{n}{ }^{\prime}\right)_{*}(\quad)
$$

defined by

$$
\pi_{12}^{P_{i}}=1+s_{P_{i} P_{i}}^{E_{i}} \delta_{P_{i}}=1+s_{i i}, \delta_{i}
$$

where 1 means the identity map and $E_{i}=E_{P_{i}}$ means a Morava's manifold for $P_{i}$. (See $\left.\S 2\right)$.

Lemma 3.5. Let $\mathscr{S}_{n-2}^{(i j)}=\left\{P_{1}, \cdots, \widehat{P}_{i}, \cdots, \widehat{P}_{j}, \cdots, P_{n}\right\}$ be the 
subsequence of $\mathscr{S}_{n}$ which is obtained from $\mathscr{S}_{n}$ by deleting its two members $P_{i}$ and $P_{j}, i \neq j$. Then we have

$$
s_{P_{i} P_{i}, \circ} S_{P_{j} P_{j^{\prime}}}=-s_{P_{j} P_{j^{\prime}}} \circ S_{P_{i} P_{i}}
$$

as homomorphisms: $\quad \operatorname{MU}\left(\mathscr{S}_{n-2}^{(i j)}+\mathscr{S}_{n}^{\prime}\right)_{*}(\quad) \rightarrow M U\left(\mathscr{S}_{n}+\mathscr{S}_{n}^{\prime}\right)_{*}(\quad)$.

Proof. Take an $\left(\mathscr{S}_{n-2}^{(i j)}+\mathscr{S}_{n}{ }^{\prime}\right)$-manifold $C$. It will be sufficient to show that $s_{P_{i} P_{i}} s_{P_{j} P_{f^{\prime}}} C$ is isomorphic to $-s_{P_{j} P_{j^{\prime}}} s_{P_{i} P_{i^{\prime}}} C$ as an $\left(\mathscr{S}_{n}+\mathscr{S}_{n}^{\prime}\right)$ manifold.

By $(2 \cdot 3)^{\prime}$, we see

$$
\begin{aligned}
& s_{P_{i} P_{i}} s_{P_{j} P_{j}} C=I_{1} \times P_{i} \times\left(I_{2} \times P_{j} \times C \cup E_{j} \times C\left(P_{j}^{\prime}\right)\right) \\
& \cup E_{i} \times\left(-I_{2} \times P_{j} \times C\left(P_{i}{ }^{\prime}\right) \cup E_{j} \times C\left(P_{i}{ }^{\prime} P_{j}{ }^{\prime}\right)\right)
\end{aligned}
$$

and, on the other hand, exchanging the order of pastings we have

$$
\begin{aligned}
s_{P_{j} P^{\prime}} & s_{P_{i} P_{i}}, C=I_{2} \times P_{j} \times\left(I_{1} \times P_{i} \times C \cup E_{i} \times C\left(P_{i}^{\prime}\right)\right) \\
& \cup E_{j} \times\left(-I_{1} \times P_{i} \times C\left(P_{j}^{\prime}\right) \cup E_{i} \times C\left(P_{j}^{\prime} P_{i}^{\prime}\right)\right) \\
= & \left\{I_{2} \times P_{j} \times I_{1} \times P_{i} \times C \cup-E_{j} \times I_{1} \times P_{i} \times C\left(P_{j}^{\prime}\right)\right\} \\
& \cup\left\{I_{2} \times P_{j} \times E_{i} \times C\left(P_{i}^{\prime}\right) \cup E_{j} \times E_{i} \times C\left(P_{j}^{\prime} P_{i}^{\prime}\right)\right\} .
\end{aligned}
$$

Comparing this expression with (3.6), we have an isomorphism

$$
\psi: s_{P_{i} P_{i}}, s_{P_{j} P^{\prime}}, C \approx-s_{P_{j} P^{\prime}} s_{P_{i} P_{i}} C
$$

which is induced by the twisting maps:

$$
\begin{aligned}
& \left(I_{1} \times P_{i}\right) \times\left(I_{2} \times P_{j}\right) \rightarrow\left(I \times P_{j}\right) \times I_{1} \times P_{i}, \\
& \left(I_{1} \times P_{i}\right) \times E_{j} \rightarrow E_{j} \times\left(I_{1} \times P_{i}\right), \quad E_{i} \times\left(I_{2} \times P_{j}\right) \rightarrow\left(I_{2} \times P_{j}\right) \times E_{i},
\end{aligned}
$$

and

$$
E_{i} \times E_{j} \rightarrow E_{j} \times E_{i}
$$

on the four pairs of corresponding parts in (3.6) and $(3 \cdot 7)$.

Proposition 3.9. The endomorphisms $\pi_{12}^{P_{i}}$ in (3.4) satisfy

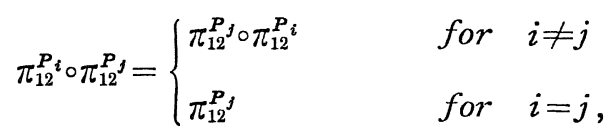


that $i s, \pi_{12}^{P_{i}}, i=1,2, \cdots, n$, are mutually commutable idempotent endomorphisms of the group $M U\left(\mathscr{S}_{n}+\mathscr{S}_{n}{ }^{\prime}\right) *(\quad)$.

Proposition 3.10. We have

i)

$$
\begin{aligned}
& \delta_{P_{i}} \circ \pi_{12}^{P_{j}}= \begin{cases}\pi_{12}^{P_{j} \circ \delta_{P_{i}}} & \text { for } i \neq j \\
0 & \text { for } i=j,\end{cases} \\
& \pi_{12}^{P_{i} \circ s_{P_{j} P_{j^{\prime}}}}= \begin{cases}s_{P_{j} P_{j^{\prime}}} \circ \pi_{12}^{P_{i}} & \text { for } i \neq j \\
0 & \text { for } i=j,\end{cases}
\end{aligned}
$$

and

$$
\operatorname{Im} \pi_{12}^{P_{i}}=\operatorname{Ker} \delta_{P_{i}}=\operatorname{Im} \gamma_{P_{i}}
$$

Proposition 3.11. Let $\gamma: M U\left(\mathscr{S}_{n}{ }^{\prime}\right)_{*}(\quad) \rightarrow M U\left(\mathscr{S}_{n}+\mathscr{S}_{n}{ }^{\prime}\right)_{*}(\quad)$ be the canonical monomorphism defined by the composition $\gamma=\gamma_{P_{1}} \cdots \gamma_{P_{n}}$ of the monomorphisms $\gamma_{P_{i}}$ (See Proposition 2.5). Then we have a direct sum decomposition of the group $M U\left(\mathscr{S}_{n}+\mathscr{S}_{n}^{\prime}\right)_{*}(\quad)$ :

$$
M U\left(\mathscr{S}_{n}+\mathscr{S}_{n}^{\prime}\right)_{*}(\quad)=\operatorname{Im} \gamma \oplus\left(\sum_{i=1}^{n} \operatorname{Im} s_{P_{i} P_{i_{i}}}^{E_{i}}\right)
$$

where $s_{P_{i} P_{i}^{\prime}}: \operatorname{MU}\left(\mathscr{S}_{n-1}^{(i)}+\mathscr{S}_{n}^{\prime}\right)_{*}(\quad) \rightarrow M U\left(\mathscr{S}_{n}+\mathscr{S}_{n}^{\prime}\right)_{*}(\quad)$.

Moreover, we have

ii) $\operatorname{Im} \gamma=\operatorname{Im} \pi_{\boldsymbol{E}}, \quad \operatorname{Ker} \pi_{\boldsymbol{E}}=\sum_{i} \operatorname{Im} s_{P_{i} P_{i^{\prime}}}, \quad \pi_{\boldsymbol{E}}=\pi_{12}^{P_{1}} \cdots \pi_{12}^{P^{n}}$, where $\boldsymbol{E}=\left\{E_{1}, \cdots, E_{n}\right\}$ is the system of Morava's manifolds $E_{i}$.

Proof of the above three propositions. Propositions 3.9 and 3.10 follow from Lemma 3.5, Propositions 2.5 and 2.7 by easy calculations.

As for Proposition 3.11, the first half i) is obtained by iterated uses of Propositions 2.5 and $2.7, \mathrm{i}$ ). To prove the second half, we first note that

$$
\pi_{12}^{P_{i}} \gamma_{P_{j}}= \begin{cases}\gamma_{P_{j}} \pi_{12}^{P_{i}} & \text { for } i \neq j \\ \gamma_{P_{j}} & \text { for } i=j .\end{cases}
$$

Using this and Proposition 3.10, ii), iii), we conclude $\pi_{\boldsymbol{E}} \mid \operatorname{Im} \gamma=i d$, and

$$
\operatorname{Ker} \pi_{\boldsymbol{E}}=\sum_{i=1}^{n} \operatorname{Im} s_{P_{i} P_{i^{\prime}}}^{E_{i}}
$$


Thus we have proved the assertion ii).

Now, we have come to introduce multiplications in the bordism theory $\operatorname{MU}\left(\mathscr{S}_{n}\right)_{*}(\quad)$.

Let $(A, f)$ and $(B, g)$ be representatives of elements of $\operatorname{MU}\left(\mathscr{S}_{n}\right)_{a}$ $(X, Y)$ and $M U\left(\mathscr{S}_{n}^{\prime}\right)_{b}(V, W)$ respectively. Let $(A \times B, f \times g)$ be the cross product of $(A, f)$ and $(B, g)$, which represents an element of $M U$ $\left(\mathscr{S}_{n}+\mathscr{S}_{n}^{\prime}\right)_{a+b}(X \times V, Y \times V \cup X \times W)$.

Let

$$
r: M U\left(\mathscr{S}_{n}^{\prime}\right)_{a+b}(\quad) \rightarrow M U\left(\mathscr{S}_{n}+\mathscr{S}_{n}^{\prime}\right)_{a+b}(\quad)
$$

and

$$
\pi_{\boldsymbol{E}}: \operatorname{MU}\left(\mathscr{S}_{n}+\mathscr{S}_{n}^{\prime}\right)_{a+b}(\quad) \rightarrow \operatorname{MU}\left(\mathscr{S}_{n}+\mathscr{S}_{n}^{\prime}\right)_{a+b}(\quad)
$$

be as in Proposition 3.11.

Then, by Proposition 3.11, ii), there is a singular $\mathscr{S}_{n}^{\prime}$-manifold $\left(A \cdot{ }_{\boldsymbol{E}} B, f \cdot{ }_{\boldsymbol{E}} g\right)$ in $(X \times V, Y \times V \cup X \times W)$ such that

$$
\gamma\left(A \cdot{ }_{\boldsymbol{E}} B, f \cdot{ }_{\boldsymbol{E}} g\right)=\pi_{\boldsymbol{E}}(A \times B, f \times g),
$$

or

$$
\left[A \cdot{ }_{\boldsymbol{E}} B, f \cdot{ }_{\boldsymbol{E}} g\right]=\left[\gamma^{-1} \circ \pi_{\boldsymbol{E}}(A \times B, f \times g)\right],
$$

where $\boldsymbol{E}=\left\{E_{1}, \cdots, E_{n}\right\}$ is the system of Morava's manifolds $E_{i}$ for $P_{i}$ which were used in defining $\pi_{12}^{P_{i}}\left(\right.$ See $\left.(3 \cdot 4)^{\prime}\right)$.

The bordism class $\left[A \cdot{ }_{\boldsymbol{E}} B, f \cdot{ }_{\boldsymbol{E}} g\right]$ depends on the choices of the system $\boldsymbol{E}$ of Morava's manifolds, but, for a fixed $\boldsymbol{E}$, it is uniquely determined from the given bordism classes $[A, f]$ and $[B, g]$.

Thus we have defined a bilinear map

$$
\begin{aligned}
\mu_{\boldsymbol{E}}: \operatorname{MU}\left(\mathscr{S}_{n}\right)_{a}(X, Y) \times M U\left(\mathscr{S}_{n}^{\prime}\right)_{b}(V, W) \\
\rightarrow M U\left(\mathscr{S}_{n}^{\prime}\right)_{a+b}(X \times V, Y \times V \cup X \times W)
\end{aligned}
$$

or a linear map

$$
\begin{aligned}
\mu_{E}: M U & \left(\mathscr{S}_{n}\right)_{a}(X, Y) \otimes M U\left(\mathscr{S}_{n}\right)_{b}(V, W) \\
& \rightarrow M U\left(\mathscr{S}_{n}\right)_{a+b}(X \times V, Y \times V \cup X \times W)
\end{aligned}
$$

by putting

$$
\mu_{\boldsymbol{E}}([A, f],[B, g])=\left[A \cdot{ }_{\boldsymbol{E}} B, f \cdot{ }_{\boldsymbol{E}} g\right]
$$


Theorem 3.14. Let $\mathscr{S}_{n}=\left\{P_{1}, \cdots, P_{n}\right\}$ be a singularity class.

Then, for any choice of system $\boldsymbol{E}=\left\{E_{1}, \cdots, E_{n}\right\}$ of Morava's manifolds $E_{i}$ one for each $P_{i}$, there exists a natural multiplication $\mu_{\boldsymbol{E}}$ in the bordism theory $M U\left(\mathscr{S}_{n}\right)_{*}(\quad)$ :

$$
\begin{aligned}
\mu_{\boldsymbol{E}}: M U & \left(\mathscr{S}_{n}\right)_{n}(X, Y) \otimes M U\left(\mathscr{S}_{n}\right)_{b}(V, W) \\
\rightarrow & M U\left(\mathscr{S}_{n}\right)_{a: b}(X \times V, Y \times V \cup X \times W)
\end{aligned}
$$

which has the bilateral unit $1 \in M U\left(\mathscr{S}_{n}\right)_{0}(p t)$, the canonical image of the unit element of the ring $M U_{*}(p t)$. Further the multiplication $\mu_{\boldsymbol{E}}$ is admissible in the following sense (cf. Araki-Toda [2]);

i) $\mu_{\boldsymbol{E}}$ is compatible with the usual multiplication by elements of $M U_{*}(p t)$.

ii) For the boundary homomorphism $\partial_{0}$, we have

$$
j_{*} \partial_{0} \mu_{\boldsymbol{E}}(x \otimes y)=j_{1^{*}} \mu_{\boldsymbol{E}}\left(\partial_{0} x \otimes y\right)+(-1)^{\operatorname{dim} x} j_{2^{*}} \mu_{\boldsymbol{E}}\left(x \otimes \partial_{0} y\right)
$$

zeluere

$$
\begin{aligned}
& (Y \times V, Y \times W) \\
& (Y \times V \cup X \times W, \phi) \stackrel{j}{\longrightarrow}\left(Y \times V \cup X \times \stackrel{j_{1}}{\downarrow} W, Y \times W\right) \\
& j_{2} \uparrow \\
& (X \times W, Y \times W)
\end{aligned}
$$

are the natural inclusion maps.

iii) For the Bockstein operator $\delta_{i}$, we have

$$
\gamma_{i} \delta_{i} \ell_{\boldsymbol{E}}(x \otimes y)=\mu_{\boldsymbol{E}}\left(\gamma_{i} \delta_{i} x \otimes y\right)+(-1)^{\operatorname{dim} x} \mu_{\boldsymbol{E}}\left(x \otimes \gamma_{i} \delta_{i} y^{y}\right), \text { for } i=1, \cdots n,
$$

that is, the operations $\gamma_{i} \hat{0}_{i}$ are derivations.

iv) For $x, y \in M U\left(\mathscr{S}_{n-1}^{(i)}\right)_{*}(\quad)$, we have

$$
\gamma_{i} \mu_{\boldsymbol{E}^{(i)}}(x \otimes y)=\mu_{\boldsymbol{E}}\left(\gamma_{i} x \otimes \gamma_{i} y\right)
$$

where $\boldsymbol{E}^{(i)}=\left\{E_{1}, \cdots \widehat{E}_{i} \cdots E_{n}\right\}=\boldsymbol{E}-\left\{E_{i}\right\}$,

$\mathrm{v})$ If one of $x, y, z$ is the image of an element of $M U_{*}(p t)$, then

$$
\left.\mu_{\boldsymbol{E}}((x \otimes y) \otimes z)\right)=\mu_{\boldsymbol{E}}(x \otimes(y \otimes z)) .
$$

Proof. $\mu_{\boldsymbol{E}}(1 \otimes x)=x$ follows directly from the definition of $\mu_{\boldsymbol{E}}$. To calculate $\mu_{\boldsymbol{E}}(x \otimes 1)$, take a representative $(A, f)$ of an element $x$ of 
$\operatorname{MU}\left(\mathscr{S}_{n}\right)_{a}(X, Y)$. Note that

$$
\begin{gathered}
\pi_{12}^{i}((A, f) \times 1)=(A, f)+s_{i i^{\prime}}, \delta_{i}(A, f)=(A, f)+(I \times P \times A(i), \\
f(i) \circ p r) \sim \sigma_{i i^{\prime}}^{*}(A, f) \text { in } M U\left(\mathscr{S}_{n}+\mathscr{S}_{n}^{\prime}\right)_{*}(X, Y) .
\end{gathered}
$$

Since $\pi_{12}^{i} \sigma_{j j^{\prime}}^{*}=\sigma_{j j^{\prime}}^{*}, \pi_{12}^{i}$ for $i \neq j$, we have

$$
\pi_{\boldsymbol{E}}(x \times 1)=\pi_{12}^{n} \circ \cdots \circ \pi_{12}^{1}(x \times 1)=\sigma_{n n^{\prime}}^{*}, \cdots \sigma_{11^{\prime}}^{*}(x \times 1)=1 \times x^{\prime}=\gamma\left(x^{\prime}\right)
$$

where $x^{\prime}$ denotes a copy of $x$ in $M U\left(\mathscr{S}_{n}^{\prime}\right)_{a}(X, Y)$ identified with $M U$ $\left(\mathscr{S}_{n}\right)_{a}(X, Y)$. It follows that

$$
\mu_{\boldsymbol{E}}(x \otimes 1)=\gamma^{-1} \cdot \pi_{\boldsymbol{E}}(x \times 1)=x^{\prime} .
$$

Similar argument applies to the case when the unit 1 is replaced by any element [M] of $M U_{*}(p t)$. The assertions i), ii), iv) and v) follows then easily and we will omit their proofs. For the proof of iii), we deduce the following equations:

$$
\begin{aligned}
& r \gamma_{i^{\prime}}, \delta_{i^{\prime}} \mu_{\boldsymbol{E}}=\gamma_{i^{\prime}} \delta_{i^{\prime}}, \pi_{\boldsymbol{E}}=\pi_{\boldsymbol{E}}{ }^{(i)} \gamma_{i^{\prime}}, \delta_{i}, \pi_{12}^{i}=\pi_{\boldsymbol{E}}{ }^{(i)} \gamma_{i^{\prime}}\left(\delta_{i^{\prime}}+\sigma_{i i^{\prime}}^{*} \delta_{i}\right) \\
& =\pi_{\boldsymbol{E}}{ }^{(i)} \gamma_{i^{\prime}}, \delta_{i^{\prime}}+\pi_{\boldsymbol{E}}{ }^{(i)} \bar{\sigma}_{i i^{\prime}}^{*} \gamma_{i} \delta_{i}, \\
& \gamma \mu_{\boldsymbol{E}}\left(\gamma_{i} \delta_{i} \otimes 1\right)=\pi_{\boldsymbol{E}}{ }^{(i)} \gamma_{i} \delta_{i},
\end{aligned}
$$

and

$$
\begin{aligned}
\gamma \mu_{\boldsymbol{E}}\left(1 \otimes \gamma_{i} \delta_{i}\right) & =\pi_{\boldsymbol{E}} \gamma_{i^{\prime}}, \delta_{i^{\prime}}=\pi_{\boldsymbol{E}}{ }^{(i)} \pi_{12}^{i} \gamma_{i^{\prime}}, \delta_{i^{\prime}} \\
& =\pi_{\boldsymbol{E}}{ }^{\left({ }^{(i)}\right.} \gamma_{i^{\prime}}, \delta_{i^{\prime}}+\pi_{\boldsymbol{E}}{ }^{(i)} s_{i i^{\prime}} \gamma_{i^{\prime}}, \delta_{i} \delta_{i^{\prime}},
\end{aligned}
$$

where $\pi_{\boldsymbol{E}}^{(i)}=\pi_{12}^{n} \cdots \pi_{12}^{\hat{i}} \cdots \pi_{12}^{1}$ is the composition of $\pi_{12}^{j}, j \neq i$. Using Proposition 2.13 and the fact that $\sigma_{i i^{\prime}}^{*}=i d$ on $M U\left(\mathscr{S}_{n-2}^{\left(i i^{\prime}\right)}\right)_{*}()$, and comparing the right hand sides of the above equations, we have

$$
\begin{aligned}
r \gamma_{i}, \delta_{i^{\prime}}, \mu_{\boldsymbol{E}} & -\gamma \mu_{\boldsymbol{E}}\left(\gamma_{i} \delta_{i} \otimes 1\right)-\gamma \mu_{\boldsymbol{E}}\left(1 \otimes \gamma_{i} \delta_{i}\right) \\
& =\pi_{\boldsymbol{E}}{ }^{(i)}\left(\bar{\sigma}_{i i^{\prime}}^{*} \gamma_{i} \delta_{i}-\gamma_{i} \delta_{i}-s_{i i^{\prime}}, \gamma_{i}, \delta_{i} \delta_{i^{\prime}}\right) \\
& =-\pi_{\boldsymbol{E}}{ }^{(i)} s_{i i^{\prime}}\left(\sigma_{i i^{\prime}}^{*} \delta_{i}, \gamma_{i} \delta_{i}+\gamma_{i^{\prime}}, \delta_{i} \delta_{i^{\prime}}\right)=0 .
\end{aligned}
$$

This proves the assertion iii) and the proof of the theorem is completed.

\section{§4. Commutativity}

Let $\mathscr{S}_{n}^{\prime}$ be a copy of a singularity class $\mathscr{S}_{n}$. Recall that in 
$M U\left(\mathscr{S}_{n}+\mathscr{S}_{n}^{\prime}\right)_{*}(\quad)$ we have the involutions

$$
\bar{\sigma}_{i}{ }^{*}=\left(\overline{i, i^{\prime}}\right)^{*}: \operatorname{MU}\left(\mathscr{S}_{n}+\mathscr{S}_{n}^{\prime}\right)_{*}(\quad) \rightarrow \operatorname{MU}\left(\mathscr{S}_{n}+\mathscr{S}_{n}^{\prime}\right)_{*}(\quad)
$$

and also we have the isomorphisms

$$
\underline{\sigma}_{i}^{*}=\left(\underline{i, i^{\prime}}\right)^{*}: M U\left(\mathscr{S}_{n}+\mathscr{S}_{n}^{\prime}\right) *(\quad) \rightarrow M U\left(\sigma_{i}\left(\mathscr{S}_{n}+\mathscr{S}_{n}^{\prime}\right)\right) *(\quad) \text {. }
$$

These are related in the following forms

$$
\bar{\sigma}_{i}^{*}={ }^{\circ} \underline{\sigma}_{i}^{*}
$$

where $c$ is the obvious identification $\operatorname{MU}\left(\mathscr{S}_{n}+\mathscr{S}_{n}{ }^{\prime}\right)_{*}() \equiv M U\left(\sigma_{i}\left(\mathscr{S}_{n}\right.\right.$ $\left.\left.+\mathscr{S}_{n}^{\prime}\right)\right)_{*}()$. (See $\left.(2 \cdot 12)-(2 \cdot 12)^{\prime \prime}.\right)$

In order to check commutativity for the product $x \cdot{ }_{E} y, x \in M U\left(\mathscr{S}_{n}\right)_{a}$ $(X), y \in M U\left(\mathscr{S}_{n}^{\prime}\right)_{b}(Y)$, we consider the following diagram

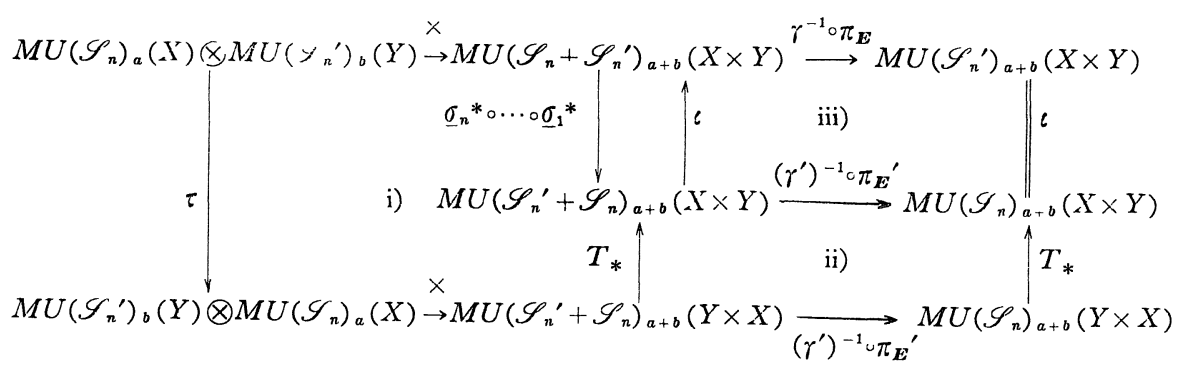

where $\tau(x \otimes y)=(-1)^{a b} y \otimes x$ and $T: Y \times X \rightarrow X \times Y$ is the twisting map. (See 3.11 for $\gamma, \pi_{\boldsymbol{E}}$.)

Lemma 4.5. The squares i), ii) and iii) in (4.4) are all commutative.

Proof. The commutativities of ii) and iii) are trivial.

In order to prove the commutativity of $\mathrm{i})$, take representatives $(A, f)$ and $(B, g)$ of $x \in M U\left(\mathscr{S}_{n}\right)_{a}(X)$ and $y \in M U\left(\mathscr{S}_{n}^{\prime}\right)_{b}(Y)$ respectively.

Then we can define an isomorphism between $\left(\mathscr{S}_{n}{ }^{\prime}+\mathscr{S}_{n}\right)$-manifolds in $X \times Y$ :

$$
\tau^{\prime}: \underline{\sigma}^{*}(A \times B, f \times g) \rightarrow\left((-1)^{a b} B \times A, T \circ(g \times f)\right)
$$




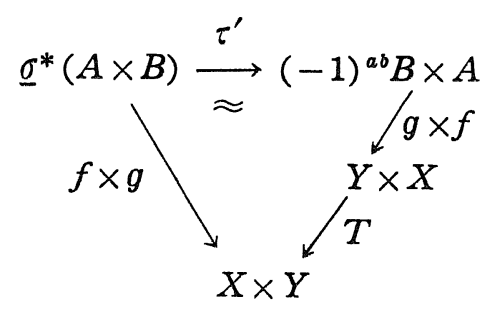

where $\underline{\sigma}^{*}=\underline{\sigma}_{n}^{*} \circ \cdots \circ \underline{\sigma}_{1}^{*}$ and $\tau^{\prime}$ is induced from the exchange of factors of the original cartesian product $A \times B$.

This means the commutativity of the diagram i) in (4.4).

From Lemma 4.5, it follows

$$
\begin{aligned}
& (-1)^{a b} T_{*}\left(y \cdot E_{E} x\right)=(-1)^{a b} T_{*}\left(\gamma^{\prime}\right)^{-1} \circ \pi_{\mathbb{E}^{\prime}}(y \times x) \\
& =(-1)^{a b}\left(\gamma^{\prime}\right)^{-1} \circ \pi_{\boldsymbol{E}^{\prime}} \circ T_{*}(y \times x)=\left(\gamma^{\prime}\right)^{-1} \circ \pi_{\boldsymbol{E}} \underline{\sigma}^{*}(x \times y) \\
& =\left(\gamma^{-1}\right) \circ \pi_{\boldsymbol{E}^{\circ}} \bar{\sigma}^{*}(x \times y)
\end{aligned}
$$

On the other hand, we can compute in the following form. By Prop. 2.13 ii), we have

$$
\bar{\sigma}_{i}^{*}(x \times y)=x \times y+s_{P_{i} P_{i^{\prime}} \bar{\delta}_{P_{i}}}^{E_{i}}\left(1-\bar{\sigma}_{i}{ }^{*}\right)(x \times y)+\alpha\left(E_{P_{i^{\prime}}}\right) \times \delta_{P_{i}} \delta_{P_{i^{\prime}}}(x \times y)
$$

or simply writing,

$$
\begin{gathered}
\bar{\sigma}_{i}{ }^{*}(x \times y)=x \times y+\alpha\left(E_{i}{ }^{\prime}\right) \times \delta_{i i^{\prime}}(x \times y)\left(\bmod \operatorname{Im} s_{P_{i} P_{i^{\prime}}}\right) \\
\text { for } \quad i=1, \cdots, n .
\end{gathered}
$$

In virtue of the commutativities

$$
\bar{\sigma}_{i}^{*} s_{P_{j} P_{j^{\prime}}}=s_{P_{j} P_{j^{\prime}}} \bar{\sigma}_{i}^{*} \text { for } i \neq j,
$$

we deduce, from (4.8), inductively

$$
\begin{aligned}
& \bar{\sigma}^{*}(x \times y)=\bar{\sigma}_{n}^{*} \cdots \bar{\sigma}_{1}^{*}(x \times y) \equiv x \times y \\
& +\sum_{k=1}^{n} \sum_{1 \leqq i_{1}<\cdots<i_{k} \leqq n} \alpha\left(E_{i_{1}^{\prime}}\right) \times \cdots \times \alpha\left(E_{i_{k^{\prime}}}\right) \times{\delta_{i_{1} i_{1} i_{2} i_{2} i^{\prime} \cdots i_{k} i_{k^{\prime}}}}(x \times y) \\
& \left(\bmod \sum_{i=1}^{n} \operatorname{Im} s_{P_{i} P_{i^{\prime}}}\right)
\end{aligned}
$$

or by using 3.11 , ii),

$(4 \cdot 11) \quad \bar{\sigma}^{*}(x \times y) \equiv x \times y$ 


$$
+\sum_{k=1}^{n} \varepsilon_{k} \sum_{1 \leqq i_{1}<\cdots<i_{k} \leqq n} \delta_{i_{1} \cdots i_{k}} x \times\left(\alpha\left(E_{i_{1}}\right) \times \cdots \times \alpha\left(E_{i_{k}}\right) \times \delta_{i_{1} \cdots i_{k} \cdot}{ }^{\prime}\right)^{\prime}
$$

$\left(\bmod \operatorname{Ker} \pi_{\boldsymbol{E}}\right)$

where $\varepsilon_{k}=(-1)^{a+(a-1)+\cdots+(a-k+1)}$.

Applying $\gamma^{-1} \circ \pi_{\boldsymbol{E}}$ to the both sides of (4.11) and comparing (4.7), we have

Theorem 4.12. For any singularity class $\mathscr{S}_{n}$ of weakly complex manifolds, the exterior multiplication $\mu_{\boldsymbol{E}}(x \otimes y)=x \cdot{ }_{\boldsymbol{E}} y$ in $\operatorname{MU}\left(\mathscr{S}_{n}\right)_{*}$ ( ) satisfies

$$
\begin{aligned}
& (-1)^{a b} T_{*}\left(y \cdot{ }_{E} x\right)-x \cdot{ }_{E} y \\
& \quad=\sum_{k=1}^{n} \varepsilon_{k} \sum_{1 \leqq i_{1}<\cdots<i_{k} \leqq n} \delta_{i_{1} \cdots i_{k}} x \cdot{ }_{\boldsymbol{E}}\left(\alpha\left(E_{i_{1}}\right) \times \cdots \times \alpha\left(E_{i_{k}}\right) \times \delta_{i_{1} \cdots i_{k}} y\right)
\end{aligned}
$$

where $\varepsilon_{k}=(-1)^{a+(a-1)+\cdots+(a-k+1)}, a=\operatorname{dim} x$.

\section{§5. Associativity}

We shall first deal with the case $n=1, \mathscr{S}_{1}=\{P\}$. For convenience, put $P=P^{\prime}=P^{\prime \prime}$ and consider the group $M U\left(P, P^{\prime}, P^{\prime \prime}\right)_{*}(\quad)$.

The following idempotent endomorphisms

$$
\pi_{12}=\pi_{12}^{P}=1+s_{P P^{\prime}}^{E} \delta_{P}, \pi_{23}=1+s_{P^{\prime} P^{\prime \prime}}^{E} \delta_{P^{\prime}}, \pi_{13}=1+s_{P P^{\prime \prime}}^{E} \hat{O}_{P}
$$

of $M U\left(P, P^{\prime}, P^{\prime \prime}\right)_{*}(\quad)$ are similarly defined as in (3.4).

Lemma 5.1. Let $\gamma: M U\left(P^{\prime \prime}\right)_{*}() \rightarrow M U\left(P, P^{\prime}, P^{\prime \prime}\right)_{*}()$ be the canonical monomorphism defined by the composition $\gamma=\gamma_{P^{\circ}} \gamma_{P^{\prime}}$. Then we have a direct sum decomposition

j) $\quad \operatorname{MU}\left(P, P^{\prime}, P^{\prime \prime}\right) *(\quad)=\operatorname{In} \gamma \oplus \operatorname{Im} s_{P P^{\prime \prime}} \oplus \operatorname{Im} s_{P^{\prime} P^{\prime \prime}} \circ \gamma_{P}$,

and

ii) $\quad \operatorname{Im} \gamma=\operatorname{Ker} \delta_{1} \cap \operatorname{Ker} \delta_{2}\left(=\operatorname{Ker} \delta_{P} \cap \operatorname{Ker} \hat{o}_{P^{\prime}}\right)$.

Proof. This is an easy consequence of Proposition 2.5.

\section{Lemma 5.2.}


Proof. A direct calculations plus Lemma 5.1, ii) prove the lemma.

\section{Proposition 5.3.}

$$
\pi_{23} \pi_{12}=\pi_{13} \pi_{23}
$$

We will postpone the proof of this proposition.

Now let $[A, f],[B, g]$ and $[C, h]$ be elements of $M U(P)_{*}(X)$, $M U\left(P^{\prime}\right)_{*}(Y)$ and $M U\left(P^{\prime \prime}\right)_{*}(Z)$ respectively. We have products

$$
\left[A \cdot{ }_{E} B, f \cdot{ }_{E} g\right] \text { in } M U\left(P^{\prime}\right)_{*}(X \times Y) \text {, }
$$$$
\left[B \cdot{ }_{E} C, g \cdot{ }_{E} h\right] \text { in } M U\left(P^{\prime \prime}\right)_{*}(Y \times Z), \quad\left[\left(A \cdot{ }_{E} B\right) \cdot{ }_{E} C,\left(f \cdot{ }_{E} g\right) \cdot{ }_{E} h\right]
$$

and

$$
\left[A \cdot_{E}\left(B \cdot{ }_{E} C\right), f \cdot_{E}\left(g \cdot{ }_{E} h\right)\right] \text { in } M U\left(P^{\prime \prime}\right)_{*}(X \times Y \times Z) .
$$

For simplicity, we will omit the mappings in the bordism classes and only deal with manifolds.

In $M U\left(P, P^{\prime}, P^{\prime \prime}\right)_{*}(X \times Y \times Z)$, we have

$$
\begin{aligned}
& \gamma\left[\left[A \cdot{ }_{E} B\right] \cdot{ }_{E} C\right]=\gamma_{P} \gamma_{P},\left[\left(A \cdot{ }_{E} B\right) \cdot{ }_{E} C\right]=\gamma_{P} \pi_{23}\left[\left(A \cdot{ }_{E} B\right) \times C\right] \\
& \quad=\pi_{23} \gamma_{P}\left[\left(A \cdot{ }_{E} B\right) \times C\right]=\pi_{23}\left[\pi_{12}(A \times B) \times C\right]=\pi_{23} \pi_{12}[A \times B \times C], \\
& \gamma\left[A \cdot{ }_{E}\left(B \cdot{ }_{E} C\right)\right]=\gamma_{P^{\prime}} \gamma_{P}\left[A \cdot{ }_{E}\left(B \cdot{ }_{E} C\right)\right]=\gamma_{P}, \pi_{13}\left[A \times\left(B \cdot{ }_{E} C\right)\right] \\
& \quad=\pi_{13}\left[A \times \pi_{23}(B \times C)\right]=\pi_{13} \pi_{23}[A \times B \times C] .
\end{aligned}
$$

Then, by Proposition 5.3, we obtain

Theorem 5.5. The exterior multiplication $\mu_{E}$ in $M U(P)_{*}()$ is associative:

$$
\left(x \cdot{ }_{E} y\right) \cdot{ }_{E} z=x \cdot{ }_{E}\left(y \cdot{ }_{E} z\right) .
$$

The next lemma plays a key role in the proof of Prop. 5.3.

Lemma 5.6. For $[C, h] \in M U\left(P^{\prime \prime}\right)_{*}(\quad)$, we have

$$
\left(s_{13}^{E} s_{23}^{E}+s_{23}^{E} s_{13}^{E}\right)[C, h]=\pi_{13}\left(\alpha\left(E_{3}\right) \times(13)^{*}[C, h]\right)
$$




$$
\left(=\pi_{13}(13) *\left(\alpha\left(E_{1}\right) \times[C, h]\right)\right)
$$

in $\operatorname{MU}\left(P, P^{\prime}, P^{\prime \prime}\right)_{*}()$. Here we are considering in the situation: $\mathscr{S}_{3}$ $=\left\{P, P^{\prime}, P^{\prime \prime}\right\}$ with $P=P^{\prime}=P^{\prime \prime},[C, h] \in M U\left(\mathscr{S}_{1}^{(12)}\right)_{*}(\quad) . \quad($ See $\S 2$.

\section{Proof. Construct a $\left\{P^{\prime \prime}\right\}$-manifold}

$$
W=s_{13} s_{23} C \bigcup_{\psi} s_{23} s_{13} C
$$

with identifications shown in the following diagram:

$$
\begin{aligned}
& s_{13} s_{23} C=I_{1} \times P \times \overbrace{\left(I_{2} \times P \times C \bigcup_{\partial_{3} \partial_{0}}^{\cup} E_{2} \times C(3)\right.}^{s_{23} C}) \bigcup_{\partial_{3} \partial_{0}} E_{1} \times \overbrace{\left(1_{2} \times C \cup E_{2}(1) \times C(3)\right.}^{\left(s_{23} C\right)}(3)) \\
& \left.\partial_{1}\right\rfloor \partial_{3} \partial_{0} \partial_{2} \\
& -0_{1} \times \underline{P} \times I_{2} \times P \times C \cup-0_{1} \times \underline{P} \times E_{2} \times C(3) \\
& \approx \downarrow \psi \quad I_{1} \times P \times 0_{2} \times \underline{P} \times C \cup E_{1} \times 0_{2} \times \underline{P} \times C(3) \\
& I_{2} \times P \times 0_{1} \times \underline{P} \times C \cup E_{2} \times 0_{1} \times \underline{P} \times C(3) \quad \approx \downarrow \psi \\
& -0_{2} \times \underline{P} \times I_{1} \times P \times C \cup-0_{2} \times \underline{P} \times E_{1} \times C(3) \\
& \partial_{1} \\
& s_{23} s_{13} C=I_{2} \times P \times(\underbrace{I_{1} \times P \times C \bigcup_{\partial_{3} \theta_{0}} E_{1} \times C(3)}_{s_{13} C}) \bigcup_{\partial_{s} \partial_{0}} E_{2} \times \underbrace{\left(1_{1} \times C \cup E_{1}(1) \times C(3)\right.}_{\left(s_{13} C\right)(3)})
\end{aligned}
$$

where $E_{1}, E_{2}$ are copies of the Morava manifold $E$ for $P$. The following lemma follows easily.

\section{Lemma 5.9.}

$$
s_{13} s_{23} C+s_{23} s_{13} C \sim \gamma(W)
$$

where $\gamma(W)$ denotes the $\left\{P, P^{\prime}, P^{\prime \prime}\right\}$-manifold $W$ with $\partial_{1} W=\partial_{2} W=\phi$.

Now $r(W)$ is considered as the union of two $\left\{P, P^{\prime \prime}\right\}$-manifolds $U$ and $V$ as follows:

$$
\gamma_{1}(W)=U \bigcup_{\partial_{1} \partial_{1}} V, U=U(E)_{(3)} \times C_{(1)}, \quad V=V(E)_{(13)} \times C(3)
$$

where 


$$
U(E)=\left(I_{1} \times P \times I_{2} \times P \cup E_{1} \times 1_{2}\right) \bigcup_{\psi}\left(I_{2} \times P \times I_{1} \times P \cup E_{2} \times 1_{1}\right),
$$

with identification indicated in $(5 \cdot 8)$. Here $U(E)$ is considered as a closed $\left\{P^{\prime \prime}\right\}$-manifold such that

$$
\begin{gathered}
\partial_{3} U(E)=\left(1_{1} \times \underline{P} \times I_{2} \times P \cup \underline{P} \times E_{1}(1) \times 1_{2}\right) \\
\bigcup_{\psi}\left(1_{2} \times \underline{P} \times I_{1} \times P \cup \underline{P} \times E_{2}(1) \times 1_{1}\right),
\end{gathered}
$$

$U=U(E) \times C$ can be considered as a $\left\{P, P^{\prime \prime}\right\}$-manifold:

$$
\begin{aligned}
& \partial_{1} U=U(E) \times \partial_{3} C \\
& \partial_{3} U=\partial_{3} U(E) \times C .
\end{aligned}
$$

Similarly we put

$$
V(E)=\left(I_{1} \times P \times E_{2} \cup E_{1} \times E_{2}(1)\right) \bigcup_{\psi}\left(I_{2} \times P \times E_{1} \cup E_{2} \times E_{1}(1)\right) .
$$

We consider $V(E)$ as a $\left\{P, P^{\prime \prime}\right\}$-manifold:

$$
\begin{gathered}
\partial_{1} V(E)=-\left\{\left(I_{1} \times P \times I_{2} \times P \times \underline{P} \cup E_{1} \times 1_{2} \times \underline{P}\right)\right. \\
\left.\bigcup_{\psi}\left(I_{2} \times P \times I_{1} \times P \times \underline{P} \cup E_{2} \times 1_{1} \times \underline{P}\right)\right\} \\
\partial_{3} V(E)=\left(1_{1} \times \underline{P} \times E_{2} \cup \underline{P} \times E_{1}(1) \times E_{2}(1)\right) \\
\bigcup_{\psi}\left(1_{2} \times \underline{P} \times E_{1} \cup \underline{P} \times E_{2}(1) \times E_{1}(1)\right),
\end{gathered}
$$

and $V=V(E) \times C(3)$ is the cross product of $V(E)$ and $C(3)$.

Note that

$$
\hat{o}_{1} V(E)=-U(E) \text {. }
$$

By $(5 \cdot 10)$, we have, as $\left\{P, P^{\prime \prime}\right\}$-manifolds,

$$
r_{1}(W) \sim U+V \text {. }
$$

By simple calculations, we have

$$
\begin{aligned}
{[U] } & =\pi_{13}\left[U(E)_{(3)} \times C_{(1)}\right]-s_{13}\left[U(E)_{(3)} \times C(3)\right] \\
& =\pi_{13}(13) *\left[U(E)_{(1)} \times C\right]-s_{18}\left[U(E)_{(3)} \times C(3)\right],
\end{aligned}
$$

and by using $(5 \cdot 13)$, we have

$$
[V]=\pi_{13}\left[V(E)_{(13)} \times C(3)\right]+s_{13}\left[U(E)_{(3)} \times C(3)\right] .
$$

But there is a unique element $[v(E)]$ in $M U\left(P^{\prime \prime}\right)_{*}(p t)$ such that 
$\pi_{13}[V(E) \times C(3)]=\pi_{13}[V(E)] \times[C(3)]=\gamma_{1}[v(E)] \times[C(3)]$,

and $\operatorname{dim} v(E)=3 \operatorname{dim} P+3$ is odd, therefore $[v(E)]=0$ in $M U\left(P^{\prime \prime}\right)_{*}(p t)$.

From the above, we have

$$
\gamma_{1}[W]=[U]+[V]=\pi_{13}(13) *\left[U(E)_{(1)} \times C\right] .
$$

Lemma 5.16. There is an isomorphism

$$
U(E) \approx a(E)=E \bigcup_{\varphi \times T} E
$$

(See $(2 \cdot 10)$ for $a(E)$.)

Proof. From $(5 \cdot 11)$ and $(5 \cdot 8)$, we see

$$
U(E)=E_{1} \times 1_{2} \cup\left(I_{1} \times P \times I_{2} \times P \bigcup_{\psi} I_{2} \times P \times I_{1} \times P\right) \cup E_{2} \times 1_{1} .
$$

The isomorphism in Lemma 5.16 will be visualized in the following figure:
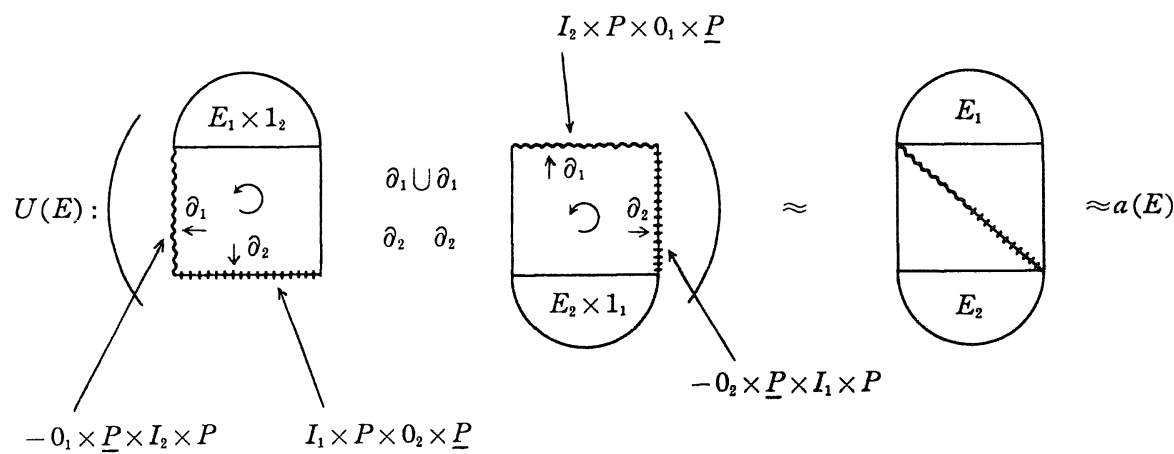

Figure 2

Now the proof of Lemma 5.6 follows from Lemma 5.9, (5.15) and Lemma 5.16.

Proof of Proposition 5.3. We shall prove, in $M U\left(P, P^{\prime}, P^{\prime \prime}\right)_{*}()$, $\pi_{23} \pi_{12}-\pi_{13} \pi_{23}=\left(1+s_{23} \delta_{2}\right)\left(1+s_{12} \delta_{1}\right)-\left(1+s_{13} \delta_{1}\right)\left(1+s_{23} \delta_{2}\right)=0$

or equivalently

$$
\left(^{*}\right) \equiv s_{12} \hat{O}_{1}-s_{18} \delta_{1}+s_{23} \delta_{2} s_{12} \hat{O}_{1}-s_{13} \delta_{1} s_{28} \delta_{2}=0 .
$$


Using Proposition 2.7, we have

$$
(*)=s_{12} \delta_{1}-s_{13} \hat{\sigma}_{1}+s_{28}(12) * \delta_{1}+s_{13} s_{23} \delta_{1} \delta_{2} .
$$

As for the first term of $(5 \cdot 18)$, we first note that

$$
s_{12}=(\overline{23}) * s_{13}(\overline{23}) *,
$$

and using Proposition 2.13 we have

$$
\begin{aligned}
s_{12} \delta_{1} & =(\overline{23}) * s_{13}(\overline{23}) * \delta_{1}=s_{13}(\overline{23}) * \delta_{1}+s_{23} \delta_{2}\left(s_{13}(\overline{23}) * \delta_{1}-s_{12} \delta_{1}\right) \\
& +\alpha(E)_{(3)} \times \delta_{2} \delta_{3} s_{13}(\overline{23}) * \delta_{1}=s_{13}(\overline{23}) * \delta_{1}-s_{23} s_{13} \delta_{2}(\overline{23}) * \delta_{1} \\
& -s_{23}(12) * \delta_{1}+\alpha(E)_{(3)} \times \delta_{2} \delta_{3} s_{13}(\overline{23}) * \delta_{1} .
\end{aligned}
$$

Putting $(5 \cdot 19)$ into $(5 \cdot 18)$, we have

$$
\begin{gathered}
\left(^{*}\right)=s_{13}\left((\overline{23})^{*}-1\right) \delta_{1}+s_{13} s_{23} \delta_{1} \delta_{2}-s_{23} s_{13} \delta_{2}(\overline{23}) * \delta_{1} \\
+\alpha(E)_{(3)} \times \delta_{2}(13) *(\overline{23})^{*} \delta_{1} .
\end{gathered}
$$

Using again Proposition 2.13, from $(5 \cdot 20)$ we have

$$
\begin{aligned}
\left(^{*}\right)=- & \left(s_{23} s_{13}+s_{13} s_{23}\right)(23) * \delta_{3} \delta_{1}+s_{13}\left(\alpha(E)_{(3)} \times \delta_{2} \delta_{3} \delta_{1}\right) \\
& +\alpha(E)_{(3)} \times(13) *(23) * \delta_{3} \delta_{1} .
\end{aligned}
$$

Then, by Lemma 5.6 ,

$$
\begin{aligned}
\left(^{*}\right)= & \pi_{13}(13)^{*}\left(\alpha(E)_{(1)} \times(23)^{*} \delta_{3} \delta_{1}\right)+s_{13}\left(\alpha(E)_{(3)} \times \delta_{2} \delta_{3} \delta_{1}\right) \\
& +\alpha(E)_{(3)} \times(13)^{*}(23)^{*} \delta_{3} \delta_{1} \\
=- & (13)^{*}\left(\alpha(E)_{(1)} \times(23)^{*} \delta_{3} \delta_{1}\right)-s_{13} \delta_{1}(13)^{*}\left(\alpha(E)_{(1)} \times(23)^{*} \delta_{3} \delta_{1}\right) \\
& +s_{13}\left(\alpha(E)_{(3)} \times \delta_{2} \delta_{3} \delta_{1}\right)+\alpha(E)_{(3)} \times(13)^{*}(23)^{*} \delta_{3} \delta_{1} \\
= & -s_{13}(13)^{*}\left(\alpha(E)_{(1)} \times \delta_{3}(23)^{*} \delta_{3} \delta_{1}\right)+s_{13}\left(\alpha(E)_{(3)} \times \delta_{2} \delta_{3} \delta_{1}\right) \\
= & -s_{13}\left(\alpha(E)_{(3)}\right) \times\left((13)^{*}(23)^{*}-1\right) \delta_{2} \delta_{3} \delta_{1} .
\end{aligned}
$$

But, $(13)^{*}(23)^{*}=i d$ on $\operatorname{Im} \delta_{2} \delta_{3} \delta_{1}$, thus we have

$$
\left(^{*}\right) \equiv \pi_{23} \pi_{12}-\pi_{13} \pi_{23}=0,
$$

and hence we have completed the proof of Proposition 5.3.

We have come to prove the associativity of the external multiplications

$$
\mu_{\boldsymbol{E}}: M U\left(\mathscr{S}_{n}\right)_{a}(X) \otimes M U\left(\mathscr{S}_{n}\right)_{b}(Y) \rightarrow M U\left(\mathscr{S}_{n}\right)_{a+b}(X \times Y)
$$


for general $n \geqq 1$.

Let $\mathscr{S}_{n}=\left\{P_{1}, \cdots, P_{n}\right\}$ be a singularity class as before and let $\mathscr{S}_{n}^{\prime}$ $=\left\{P_{1}^{\prime}, \cdots, P_{n}^{\prime}\right\}, \mathscr{S}_{n}^{\prime \prime}=\left\{P_{1}^{\prime \prime}, \cdots, P_{n}^{\prime \prime}\right\}$ be copies of $\mathscr{S}_{n}$ such that $P_{i}=P_{i}^{\prime}$ $=P_{i}^{\prime \prime}, 1 \leqq i \leqq n$.

Take up bordism classes

$x \in M U\left(\mathscr{S}_{n}\right)_{a}(X), \quad y \in M U\left(\mathscr{S}_{n}^{\prime}\right)_{b}(Y), \quad z \in M U\left(\mathscr{S}_{n}^{\prime \prime}\right)_{c}(Z)$.

Then we have the cross product ( $\$ 3$ )

$$
x \times y \times z \text { in } \operatorname{MU}\left(\mathscr{S}_{n}+\mathscr{S}_{n}^{\prime}+\mathscr{S}_{n}^{\prime \prime}\right)_{a+b+c}(X \times Y \times Z) .
$$

Choose a system $\boldsymbol{E}=\left\{E_{1}, \cdots, E_{n}\right\}$ of Marava's manifolds $E_{i}$ for $P_{i}$. Then, we have the endomorphisms

$$
\begin{gathered}
\pi_{12}^{i}=1+s_{P_{i} P_{i^{\prime}}}^{E_{i}} \delta_{P_{i}}, \pi_{23}^{i}=1+s_{P_{i^{\prime}} P_{i^{\prime \prime}}}^{E_{i}} \hat{O}_{P^{\prime}}, \pi_{13}^{i}=1+s_{P_{i} P_{i^{\prime \prime}}}^{E_{i}} \delta_{P_{i}}, \\
i=1, \cdots, n,
\end{gathered}
$$

in the group $M U\left(P_{1}, \cdots, P_{n}, P_{1}^{\prime}, \cdots, P_{n}^{\prime}, P_{1}^{\prime \prime}, \cdots, P_{n}^{\prime \prime}\right)_{*}(X \times Y \times Z)$.

Lemma 5.23. For the above endomorphisms $\pi_{12}^{i}, \pi_{23}^{i}, \pi_{13}^{i}$, we have
i) $\pi_{k l}^{i} \circ \pi_{s t}^{j}=\pi_{s t}^{j} \circ \pi_{k l}^{i} \quad$ for $\quad i \neq j$,
ii) $\pi_{23}^{i} \circ \pi_{12}^{i}=\pi_{13}^{i} \circ \pi_{23}^{i} \quad$ for $\quad i=1, \cdots, n$.

Proof. The first assertion is trivial, and the second assertion and its proof are essentially the same as those of Proposition 5.3.

Let $\gamma: M U\left(\mathscr{S}_{n}^{\prime \prime}\right)_{*}() \rightarrow M U\left(\mathscr{S}_{n}+\mathscr{S}_{n}^{\prime}+\mathscr{S}_{n}^{\prime \prime}\right)_{*}(\mathrm{)}$ be the canonical monomorphism as before. Then, by Lemma 5.23, we have

$$
\begin{aligned}
& r\left(\left(x \cdot{ }_{E} v\right) \cdot{ }_{E} z\right)=\left(\pi_{23}^{n} \circ \cdots \circ \pi_{23}^{1}\right) \circ\left(\pi_{12}^{n} \circ \cdots \circ \pi_{12}^{1}\right)(x \times y \times z) \\
& \quad=\left(\pi_{23}^{n} \circ \pi_{12}^{n}\right) \circ\left(\pi_{23}^{n-1} \circ \pi_{12}^{n-1}\right) \circ \cdots \circ\left(\pi_{23}^{1} \circ \pi_{12}^{1}\right)(x \times y \times z) \\
& \quad=\left(\pi_{13}^{n} \circ \pi_{23}^{n}\right) \circ\left(\pi_{13}^{n-1} \circ \pi_{23}^{n-1}\right) \circ \cdots \circ\left(\pi_{13}^{1} \circ \pi_{23}^{1}\right)(x \times y \times z) \\
& \quad=\left(\pi_{13}^{n} \circ \cdots \circ \pi_{13}^{1}\right) \circ\left(\pi_{23}^{n} \circ \cdots \circ \pi_{23}^{1}\right)(x \times y \times z)=\gamma\left(x \cdot{ }_{\boldsymbol{E}}\left(y \cdot{ }_{\boldsymbol{E}} z\right)\right) .
\end{aligned}
$$

Thus we have proved

Theorem 5.25. For any singularity class $\mathscr{S}_{n}$ of weakly complex 
manifolds and for any choice of the system $\boldsymbol{E}$, the exterior multiplication $\|_{\boldsymbol{E}}$ in $\operatorname{MU}\left(\mathscr{S}_{n}\right)_{*}(\quad)$ is associative.

Corollary 5.26. The dual cohomology theory $M U\left(\mathscr{S}_{n}\right){ }^{*}(\quad)$ has also associative multiplications compatible with the module structure over $M U^{*}(p t)$.

\section{§ 6. Conclusions}

Corresponding to any singularity class $\mathscr{S}_{n}=\left\{P_{1}, \cdots, P_{n}\right\}$ of closed, weakly complex manifolds and any choice of system $\boldsymbol{E}=\left\{E_{1}, \cdots, E_{n}\right\}$ of Morava's manifolds $E_{i}$ one for each $P_{i}$, we have obtained a natural multiplication

$$
\begin{gathered}
\mu_{\mathbb{E}}: M U\left(\mathscr{S}_{n}\right)_{*}(X, Y) \otimes M U\left(\mathscr{S}_{n}\right)_{*}(V, W) \rightarrow M U\left(\mathscr{S}_{n}\right)_{*}(X \times V, \\
Y \times V \cup X \times W)
\end{gathered}
$$

which is admissible (Theorem 3.14) and associative (Theorem 5.25).

And, if the obstruction classes $\alpha\left(E_{i}\right)$ or their canonical images in the coefficient group $\operatorname{MU}\left(\mathscr{S}_{n}\right)_{*}(p t)$ happen to vanish, then $\mu_{\boldsymbol{E}}$ is commutative (Theorem 4.12). We can consider such favoring situations corresponding to suitable choices of $\mathscr{S}_{n}$ and $\boldsymbol{E}$.

In the below, for convenience, we consider only the case when the singularity class $\mathscr{S}_{n}$ represents a regular sequence in $M U_{*}(p t)$.

Corollary 6.1. Let $\mathscr{S}_{n}=\left\{P_{1}, \cdots, P_{n}\right\}$ represent a regular sequence in $M U_{*}(p t)$. Then any multiplication $\mu_{\boldsymbol{E}}$ as above induces a unique ring structure in the coefficient group $M U\left(\mathscr{S}_{n}\right)_{*}(p t)$ which is canonically isomorphic to the quotient ring $M U_{*}(p t) /\left(\left[P_{1}\right], \cdots,\left[P_{n}\right]\right) . \quad(C f$. Proposition 1.13).

For the next theorem, suppose $\alpha\left(E_{i}\right) \equiv 0(\bmod 2)$ in $M U\left(\mathscr{S}_{n}\right)_{*}(p t)$ for $i=1,2, \cdots, n$. Then, by Lemmas 2.2 and 2.11, we can choose another Morava's manifold $E_{i}{ }^{\prime}$ in place of $E_{i}$ for each $i$ such that $\alpha\left(E_{i}{ }^{\prime}\right)=0$ in $M U\left(\mathscr{S}_{n}\right)_{*}(p t)$. Thus we have.

Theorem 6.2. Let $\mathscr{S}_{n}$ represent a regular sequence in $M U_{*}(p t)$. Assume that every element of $M U\left(\mathscr{S}_{n}\right)_{2 p_{i}+2}(p t), p_{i}=\operatorname{dim} P_{i}$, is divisible 
by 2 for each $i=1,2, \cdots, n$.

Then there is an external multiplication $\|_{\boldsymbol{E}}$ in the bordism theory $\operatorname{MU}\left(\mathscr{S}_{n}\right)_{*}(\quad)$, which is admissible, associative and commutative. Therefore the dual cohomology theory $\operatorname{MU}\left(\mathscr{S}_{n}\right)^{*}()$ is multiplicative and the representing spectrum $M U\left(\mathscr{S}_{n}\right)$ is a (commutative) ring spectrum.

Corollary 6.3. Assume that $\mathscr{S}_{n}$ represent a regular sequence in $M U_{*}(p t)$ and contain a member, say, $P_{1}=Z / 2 k+1$, the point set of odd number of elements. (In this case $M U\left(\mathscr{S}_{n}\right)_{*}()$ is a $(Z / 2 k+1)$ module.)

Then there is an admissible, associative and commutative multiplication in $\operatorname{MU}\left(\mathscr{S}_{n}\right)_{*}$ ( ) (In such a case we shall call the homology theory $\operatorname{MU}\left(\mathscr{S}_{n}\right)_{*}($ ) multiplicative), and so is the cohomology theory $\operatorname{MU}\left(\mathscr{S}_{n}\right)^{*}() . \quad(c f .[2],[11])$.

So far we assumed $\mathscr{S}_{n}$ is a finite sequence, but this restriction will be unnecessary and we can generalize, without difficulty, all the above argument to the case $n=\infty$. In fact, if we take an infinite sequence $\mathscr{S}=\left\{P_{1}, P_{2}, \cdots\right\}$ of closed, weakly complex manifolds as a singularity class, the corresponding homology theory $M U(\mathscr{S})_{*}()$ can be defined as the direct limit $\lim _{\longrightarrow} M U\left(\mathscr{S}_{n}\right)_{*}(\quad)$ of the sequence

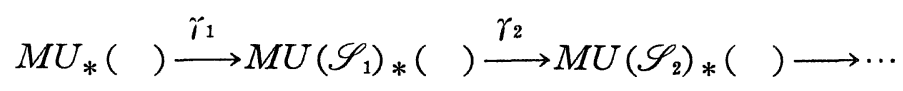

for the sections $\mathscr{S}_{n}=\left\{P_{1}, \cdots, P_{n}\right\}$ of $\mathscr{S}$. Or, we may start, from the outset, with singular $\mathscr{S}$-manifolds, just as in the case of singular $\mathscr{S}_{n^{-}}$ manifolds but for $n$ unrestricted, and proceed as usual to defining bordism theory (See Baas [4]).

Corollary 6.4 (Corollary 5.1 in [4]). If $\mathscr{S}=\left\{P_{1}, P_{2}, \cdots\right\}$ represents a generating system $\left\{x_{1}, x_{2}, \cdots\right\}$ of the polynomial algebra $M U_{*}$ $(p t)=\boldsymbol{Z}\left[x_{1}, x_{2}, \cdots\right], x_{n}=\left[P_{n}\right], \operatorname{dim} x_{n}=2 n$, then we have

$$
M U\left(\mathscr{S}^{\prime}\right)_{*}(\quad)=H_{*}(, Z)
$$

where the right hand side is the ordinary singular homology theory. 
If we take $\mathscr{S}=\left\{P_{1}, P_{3}, \cdots, P_{2 k+1}, \cdots\right\},\left[P_{2 k+1}\right]=x_{2 k+1}, k \geqq 0$, as a sequence representing $\left\{x_{1}, x_{3}, \cdots, x_{2 k+1}, \cdots\right\}$ in $M U_{*}(p t)=\mathbb{Z}\left[x_{1}, x_{2}, \cdots\right]$, then the coefficient group $M U(\mathscr{S})_{*}(p t)$ vanishes in dimensions $4 l+2(l \geqq 0)$ and is isomorphic to $Z\left[x_{2}, x_{4}, \cdots, x_{2 k}, \cdots\right]$. In such a case, we write also $M U(\mathscr{S})_{*}(\mathrm{l})=M U\left\langle x_{2}, x_{4}, \cdots, x_{2 k}, \cdots\right\rangle_{*}\left(\mathrm{)}\right.$. Since $\operatorname{dim} \alpha\left(E_{2 k+1}\right)=8 k+4$ +2 in this case, the images of $\alpha\left(E_{2 k+1}\right)$ in $M U(\mathscr{S})_{*}(p t)$ are all zero. So we have

Theorem 6.5. If $\mathscr{S}=\left\{P_{1}, P_{3}, \cdots, P_{2 k+1}, \cdots\right\}$ represents the subsequence $\left\{x_{1}, x_{3}, \cdots, x_{2 k+1}, \cdots\right\}$ of the polynomial basis of $M U_{*}(p t)=\mathbb{Z}\left[x_{1}\right.$, $\left.x_{2} \cdots\right]$, then the homology theory $M U(\mathscr{S})_{*}()=M U\left\langle x_{2}, x_{4}, \cdots, x_{2 k}, \cdots\right\rangle_{*}$ ( ) is multiplicative, and so the cohomology theory $M U(\mathscr{S})^{*}($ ).

Similarly we have

Theorem 6.6. The cohomology theory $M U\left\langle x_{2 k_{i}} \mid 1 \leqq k_{1}<k_{2}<\cdots\right\rangle^{*}()$ is multiplicative.

Fix an odd prime $p$. Taking $x_{i}=u_{i+1}, u_{p^{i}}=v_{i}, i \geqq 1$, the Hazewinkel generators of $M U_{*}(p t)$ ([16], cf. [15] and [17]), we can deduce, from [5],

Corollary 6.7. The (integral) Brown-Peterson cohomology theory $B P^{*}()=M U\left\langle v_{i} \mid i \geqq 1\right\rangle^{*}()$ for an odd prime $p$ is multiplicative and the spectrum BP is a ring spectrum. (The adjective "integral" means "not localized at $p^{\prime \prime}$.) And similarly for $B P\left\langle v_{i_{1}}, v_{i_{2}}, \cdots \mid i_{1}<i_{2}<\cdots\right\rangle^{*}($ ) and, in particular, $k\langle n\rangle^{*}()=B P\left\langle v_{n}\right\rangle^{*}()$, the connective extraordinary $K$-theory, as well $K\langle n\rangle^{*}()=\left(v_{n}\right)^{-1} k\langle n\rangle^{*}()$, the periodic cohomology after Morava ([10], [12]).

\section{References}

[1] Adams, J. F., Quillen's work of formal groups and complex cobordism, Stable Homotopy and Generalised Homology, Part II, Univ. of Chicago Press, Chicago 1974.

[2] Araki, S. and Toda, H., Multiplicative structures in $\bmod q$ cohomology theories, I, II, Osaka J. Math., 2 (1965), 71-115; 3 (1966), 81-120. 
[3] Baas, N. A., On bordism theories of manifolds with singularities, Aarhus Universitct, Preprint 31, 1969/70.

[4] - On bordism theory of manifolds with singularities, Math. Scand., 33 (1973), 279-302.

[5] Baas, N. A. and Madsen, I., On the realization of certain modules over the Steenrod algebra, Math. Scand., 31 (1972), 220-224.

[6] Baas, N. A., On formal groups and singularities in complex cobordism theory, Math. Scand., 33 (1973), 303-313.

[7] Brown, E. H. Jr. and Peterson, F. P., A spectrum whose $\boldsymbol{Z}_{p}$ cohomology is the algebra of reduced $p^{\text {th }}$ powers, Topology, 5 (1966), 149-154.

[8] Cerf, J., Topologie de certains espaces de plongements, Bull. Soc. Math. France. 89 (1961), 227-380.

[9] Jänich, K., On the classification of $O(n)$-manifolds, Math. Ann., 176 (1968), 53-76.

[10] Johnson, D. C. and Wilson, W. S., BP operations and Morava's extraordinary Ktheories, Math. Zeit., 144 (1975), 55-75.

[11] Kamata, M., On the differential $d_{3}^{p, 0}$ of $U$-cobordism spectral sequence, Osaka $J$. Math., 8 (1971), 233-241.

[12] Morava, J., Unitary cobordism and extraordinary K-theories, Columbia Univ. Preprint.

[13] Sullivan, D., Geometric Topology Seminar Notes, Princeton Univ. 1967.

[14] , Geometric periodicity and the invariants of manifolds, ManifoldsAmsterdam 1970, Lect. Notes in Math. 197, Springer 1971.

[15] Araki, S., Typical formal groups in complex cobordism and $K$-theory, Lectures in Math. 6, Kyoto University, 1973.

[16] Hazewinkel, M., A universal formal group and complex cobordism, Bull. Amer. Math. Soc., 81 (1975), 930-933.

[17] Kozma, I., Witt vectors and complex cobordism, Topology, 13 (1974), 389-394. 
